\title{
Investigation of the Sharkskin melt instability using optical Fourier analysis
}

\author{
Alex Gansen, ${ }^{1}$ Martin Řehoř ${ }^{2}$ Clemens Sill, ${ }^{3}$ Patrycja Polińska, ${ }^{3}$ Stephan Westermann, ${ }^{3}$ Jean Dheur, ${ }^{3}$ \\ Jack S. Hale, 2 Jörg Baller $\mathbb{1}^{1}$ \\ ${ }^{1}$ Physics and Materials Science Research Unit, University of Luxembourg, 162a avenue de la Faïencerie, Luxembourg, Luxembourg \\ ${ }^{2}$ Institute of Computational Engineering, University of Luxembourg, 6 Avenue de la Fonte, L-4362, Esch-sur-Alzette, Luxembourg \\ ${ }^{3}$ Goodyear Innovation Center Luxembourg, Avenue Gordon Smith, L-7750, Colmar-Berg, Luxembourg \\ Correspondence to: J. Baller (E-mail: joerg.baller@uni.lu)
}

\begin{abstract}
An optical method allowing the characterization of melt flow instabilities typically occurring during an extrusion process of polymers and polymer compounds is presented. It is based on a camera-acquired image of the extruded compound with a reference length scale. Application of image processing and transformation of the calibrated image to the frequency domain yields the magnitude spectrum of the instability. The effectiveness of the before mentioned approach is shown on Styrene-butadiene rubber (SBR) compounds, covering a wide range of silica filler content, extruded through a Göttfert capillary rheometer. The results of the image-based analysis are compared with the results from the sharkskin option, a series of highly sensitive pressure transducers installed inside the rheometer. A simplified version of the code used to produce the optical analysis results is included as supplementary material. ๑ 2019 Wiley Periodicals, Inc. J. Appl. Polym. Sci. 2019, 137, 48806.
\end{abstract}

KEYWORDS: optical analysis; rheology; rubber compound

Received 26 August 2019; accepted 6 November 2019

DOI: 10.1002/app.48806

\section{INTRODUCTION}

Melt instabilities are a critical factor limiting the maximum throughput of industrial extrusion processes. These melt instabilities appear with increasing shear rate. For a typical polymer undergoing increasing extrusion shear rates, one expects to see a smooth extrudate at low shear rates, succeeded by the sharkskin instability, followed by a transition to the stick-slip regime, and finally gross-melt-fracture. These instabilities result in extrudates of unacceptable quality for manufacturing. The three aforementioned instabilities are briefly discussed in the following paragraphs. For a full review of melt instabilities, the interested reader is referred to the following papers. ${ }^{1-3}$

The sharkskin instability, referred to as just sharkskin henceforth, is a surface instability of height far smaller than the thickness of the extrudate. When sharkskin is well developed, it manifests as periodic structure with an amplitude of a few tens to hundreds of microns over the whole extruded sample surface. Although the presence of sharkskin does not necessarily alter the physical properties of the bulk extrudate, it does lead to a change in the surface texture of the extrudate which, might prevent the adherence of two layers of the extrudate. If two layers of the extrudate need to be glued together they might not adhere properly due to sharkskin. The precise origins of sharkskin instability are still unclear. $^{1,4}$ Although many publications suggest that its origin is related to phenomenon at the die exit, Palza and Filipe ${ }^{4,5}$ showed that it can be measured throughout the whole die by using an in situ measurement technique based on piezoelectric pressure transducers. The stick-slip instability, referred to as just stick-slip henceforth, is characterized by alternating smooth and rough regions at the extrudates surface. It is accompanied by important pressure fluctuations of about $10 \%$ of the mean pressure measured by the pressure transducer in the barrel. It is still under debate if there is a direct correlation between sharkskin and stick-slip. Sharkskin and stick-slip are surface instabilities in contrast to the gross-melt instability which is characterized by the distortion of the whole extrudate and can therefore be classified as volume instability.

There are a limited number of existing methods for characterizing melt instabilities. Wilhelm et al. $^{6-9}$ developed and commercialized in conjunction with Göttfert the so-called sharkskin option to a capillary rheometer. The sharkskin option consists of the addition of a series of highly sensitive piezoelectric pressure transducers installed inside a specially designed slit die. With these sensors, it is possible to measure the pressure fluctuations 
along the die during the extrusion process. The Fourier transformation of the pressure signal allows the determination of the characteristic frequency of the melt flow instabilities. The method is very accurate and informative about the character of the melt instabilities. The drawback of this method is that a specially designed slit die with very fast and sensitive piezoelectric pressure transducers must be used. Another method to characterize the extrudate has been suggested by Viloria. ${ }^{6}$ This method numerically characterizes the contour of an extrudate extruded from a round hole capillary. The drawback of the method of is that it is limited to circular capillary exit geometries, but in many industrial processes a slit die is far more common. Other characterization techniques are microscopy observations of cross sections ${ }^{10,11}$ profilometry measurements, ${ }^{12,13}$ image analysis, ${ }^{7,8}$ and optical or scanning electron microscopy. ${ }^{9,14}$

The optical method proposed in this article is not designed to replace the sharkskin procedure. ${ }^{5,15-17}$ That method remains the best approach for understanding instabilities in highly controlled laboratory experiments where the sharkskin option is available. In fact, the sharkskin option is used as a benchmark against which the quality of the optical method is assessed. Instead, the proposed method provides reasonably accurate characterization and is suitable for use in an industrial manufacturing context or in laboratory settings where the sharkskin option is not available or simply not practical. The method suggested in this article uses a Fourier analysis of imaging data instead to characterize melt flow instability and could be used in the context of a large-scale manufacturing process independently of the shape of the dies. For polyethylene samples, Naue used a similar technique. ${ }^{15,16}$ Focusing on different image enhancement techniques, the image analysis can be significantly improved, leading to characteristic frequencies in the same order of magnitude with characteristic frequencies of the piezoelectric pressure transducer measurements for SBR compounds with varying silica content.

\section{SAMPLE PREPARATION AND MEASUREMENTS}

The polymers and compounds under investigation are based on a SBR polymer containing $27 \%$ of styrene and functionalized end chains designed to promote interaction with silica fillers. The average molecular weight is medium with $M w=310000 \mathrm{~g} \mathrm{~mol}^{-1}$ (measured with GPC relative to standard polystyrene). The silica employed is Zeosil Premium 200 MP from Solvay. The compounds have silica contents of $0,30,70$, and $112 \mathrm{phr}$ (parts per hundred rubber). The compounds are extruded at a temperature of $100^{\circ} \mathrm{C}$ at shear rates ranging between $\dot{\gamma}=10-200 \mathrm{~s}^{-1}$ as the sharkskin instability appears in this range. The measurements are carried out using the capillary rheometers Rheograph 25 and 50 from Göttfert with the sharkskin option.,15-17

Most of the measurements have been carried out with the Rheograph 50 with a slit die of a length of $30 \mathrm{~mm}$, a width of $W=5 \mathrm{~mm}$, a height of $H=0.5 \mathrm{~mm}$ and hence an aspect ratio of $W / H=10$. For this slit die, three piezoelectric pressure transducers with a sampling rate of $20 \mathrm{kHz}$ are located along the die. They are positioned 3, 15, $27 \mathrm{~mm}$ from the die entry. A sketch of the sharkskin option is represented in Figure 1. Additional measurements have been carried out with the Rheograph 25 also including the sharkskin option but with a slit die of a length of

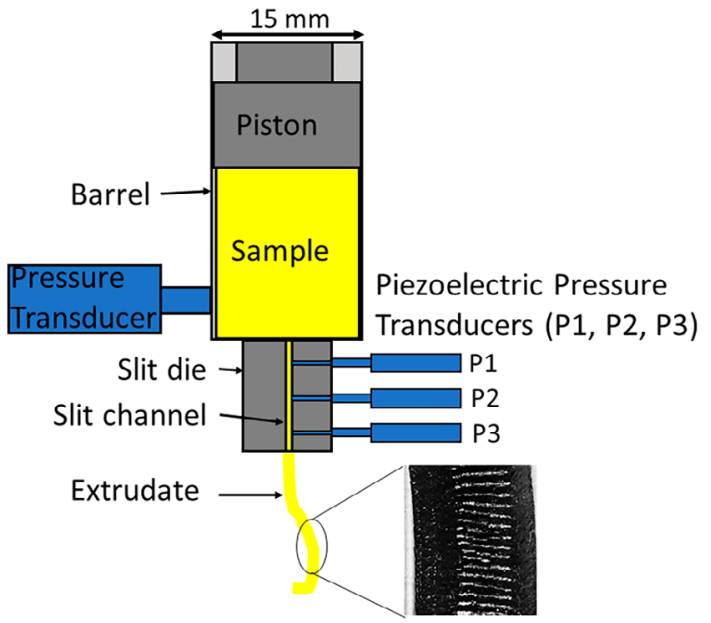

Figure 1. Göttfert sharkskin option with three piezoelectric pressure transducers located alongside the die. [Color figure can be viewed at wileyonlinelibrary.com]

$30 \mathrm{~mm}$, a width of $W=3 \mathrm{~mm}$, a height of $H=0.3 \mathrm{~mm}$ and hence an aspect ratio of $W / H=10$. This option only has one piezoelectric pressure transducer located $15 \mathrm{~mm}$ from the die entry. Before a measurement, the rubber is heated up to $100{ }^{\circ} \mathrm{C}$ for $5 \mathrm{~min}$ in the barrel of the rheometer. For a selected shear rate, the measurement with the sharkskin option is started after a constant pressure in the barrel is achieved. To measure sharkskin, a measuring time of $20 \mathrm{~s}$ has been used. ${ }^{17}$ The Fourier transform is then applied to the recorded pressure time data, allowing to characterize the specific instability.

\section{OPTICAL ANALYSIS METHOD}

For the image analysis, a photograph of the cooled extrudate if required, and, if possible the photograph should be taken under uniform lightning conditions. In this case, it should be possible to clearly distinguish the melt instability, if present, from the rest of the sample.

If this is not the case, it is possible to enhance the quality of the photograph by following the steps described in section 4. Furthermore, a length scale needs to be present in the image, for example, a ruler.

Figure 2 shows a well-developed sharkskin instability resulting from an extrusion process of unfilled SBR rubber through a slit die with height $H=0.5 \mathrm{~mm}$ and width $W=5 \mathrm{~mm}$. A digital gray scale picture can be represented as a $2 \mathrm{D}$ array of brightness values associated with each pixel. This might be represented as a function $(i, j):(\mathbb{Z}+)^{2} \rightarrow \mathbb{Z}$, where $i, j$ are the coordinates representing the $x, y$ values respectively. Before applying the Fourier transform, the $x$ axis needs to be transformed from a length into a time scale. This can easily be done using the shear rate of the extrudate at which the rubber got extruded through the capillary or via the piston speed inside the barrel. The extrusion speed $v_{\text {ext }}$ needs to be determined from the shear rate. As the piston moves with a velocity $v_{\text {piston }}$ inside the barrel it leads to a volume throughput defined as $Q_{b a r r e l}$ in the barrel. Similarly, for the capillary, $Q_{\text {capillary }}$ is the cut through surface of the capillary times 


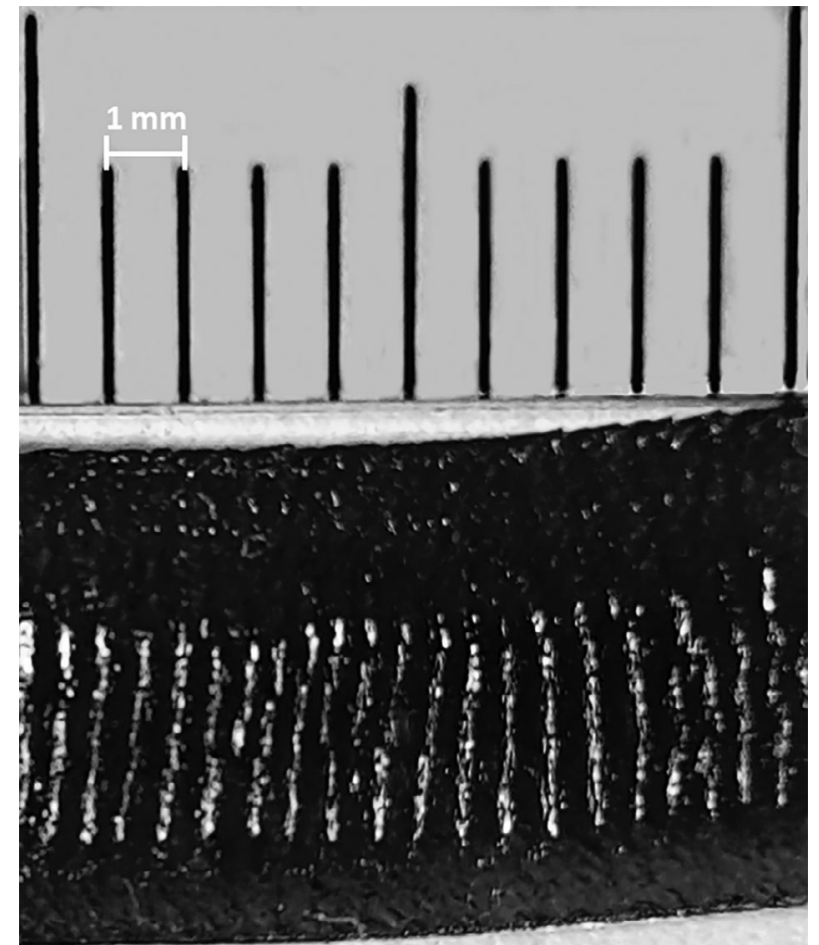

Figure 2. Image of unfilled SBR extruded at a shear rate of $\dot{\gamma}=33 \mathrm{~s}^{-1}$ displaying sharkskin.

the speed of the extrudate. If an incompressible material is assumed, as in this case, $Q_{\text {barrel }}=Q_{\text {slit }}=Q_{\text {capillary }}$. At the die exit, the flow type changes from lamellar to plug flow which affects the speed of the rubber close to the die wall

$$
\begin{gathered}
Q_{\text {barrel }}=\frac{\pi D^{2}}{4} v_{\text {piston }} \\
Q_{\text {slit }}=W H v_{\text {ext }} \\
Q_{\text {capillary }}=\frac{\pi d^{2}}{4} v_{\text {ext }}
\end{gathered}
$$

where $D$ is the diameter of the barrel, $W$ is the width and $H$ the height of the rectangular die with $W \gg H, d$ the diameter of the round hole capillary and $v_{\text {ext }}$ the velocity of the extrudate. Furthermore, the shear rate for a slit die and a round hole capillary are defined as

$$
\begin{gathered}
\dot{\gamma}_{\text {slit }}=\frac{6 Q_{\text {slit }}}{W H^{2}} \\
\dot{\gamma}_{\text {capillary }}=\frac{32 Q_{\text {capillary }}}{\pi d^{3}}
\end{gathered}
$$

Introducing eq. (2) into eq. (4) leads to

$$
\dot{\gamma}_{s l i t}=\frac{6}{H} v_{e x t}
$$

directly linking the shear rate of the slit die to the speed of the extrudate. Proceeding similarly for the capillary by introducing eq. (3) into eq. (5) the following equation is obtained

$$
\dot{\gamma}_{\text {capillary }}=\frac{8}{d} v_{\text {ext }}
$$

To illustrate the method, a slit die with dimensions $H=0.5 \mathrm{~mm}$, $W=5 \mathrm{~mm}$ and a shear rate of $\dot{\gamma}_{\text {slit }}=33 \mathrm{~s}^{-1}$ has been used. Using eq. (6) with $\dot{\gamma}_{\text {slit }}=33 \mathrm{~s}^{-1}$ and solving for $v_{\text {ext }}$ a piston speed of $v_{\text {ext }}=2.75 \mathrm{~mm} \mathrm{~s}^{-1}$ is obtained. As a length scale is associated to the sample and with the extrusion speed the extrusion time of the sample is computed. Therefore, the extension in $x$ direction in pixels is measured. The image represented in Figure 2 has $N_{x}=1776$ pixels in $x$ direction and according to the scale a length $l_{\text {ext }}=10.39 \mathrm{~mm}$. A pixel therefore has a length of $l_{\text {pixel }}=l_{\text {ext }} / N_{x}=0.0058 \mathrm{~mm}$. To convert the $x$ axis into the time domain, the time $t_{e x t}$ it takes the extrudate to be extruded, $t_{\text {ext }}=l_{\text {ext }} / v_{\text {ext }}=3.78 \mathrm{~s}$ needs to be computed. One pixel corresponds to $t_{\text {pixel }}=t_{\text {ext }} / N_{x}=0.0021 \mathrm{~s}$. From $t_{\text {pixel }}$ the Nyquist frequency required for the transformation of the time into a frequency is calculated. One pixel is recorded ever $t_{\text {pixel }}=0.0021 \mathrm{~s}$. Hence, during one second $1 / t_{\text {pixel }}=476$ pixels are recorded. This results in a Nyquist frequency of $f_{\text {Nyquist }}=\left(1 / t_{\text {pixel }}\right) / 2=238 \mathrm{~Hz}$. Combining the different relations, the Nyquist frequency can also be directly computed using

$$
f_{\text {Nyquist }}=\frac{N_{x} v_{\text {ext }}}{2 l_{\text {ext }}}
$$

\section{Frequency-Time Domain Transformation}

This section is only a small review about the Fourier transform in the ideal case of a single frequency time domain signal. Using the Fourier transform a time domain signal is converted to the frequency domain

$$
\begin{gathered}
f(t)=\frac{1}{2 \pi} \int_{\omega=-\infty}^{\infty} F(\omega) e^{i \omega t} d \omega \\
F(\omega)=\int_{t=-\infty}^{\infty} f(t) e^{-i \omega t} d t
\end{gathered}
$$

where $\omega=2 \pi f$ is the angular frequency, $f$ the frequency and $t$ the time. For a pure sinusoidal signal $f(t)=A \sin \left(\omega_{0} t\right)$ the Fourier transform becomes

$$
\begin{gathered}
F(\omega)=\int_{t=-\infty}^{\infty} A \sin \left(\omega_{0} t\right) e^{-i \omega t} d t \\
=A \pi i\left[\delta\left(\omega_{0}+\omega\right)-\delta\left(\omega_{0}-\omega\right)\right]
\end{gathered}
$$

where the delta distribution is defined as

$$
\delta(\omega)=\frac{1}{2 \pi} \int e^{i \omega t} d t
$$




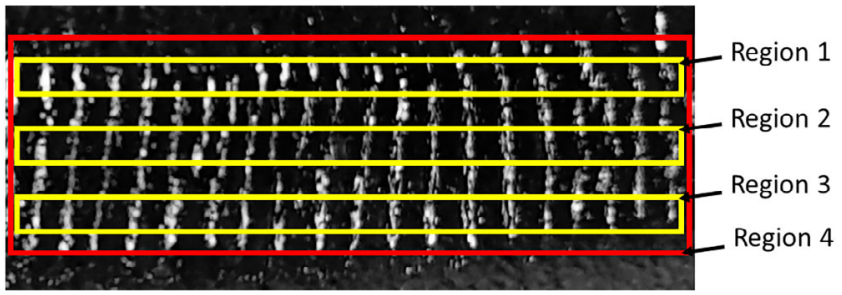

Figure 3. Visual illustration of different regions required for the Fourier analysis on unfilled SBR extrudate displaying sharkskin. Extruded at a shear rate of $\dot{\gamma}=33 \mathrm{~s}^{-1}$. [Color figure can be viewed at wileyonlinelibrary.com]

For practical applications, the Fourier transform as defined in eq. (10) cannot be applied as the integration boundaries are not infinite and as only a discretized dataset is available. Instead, the Fast Fourier Transform, a discrete version of eq. (10) which computes the transformation efficiently is employed. The result of the Fourier Transform in the ideal analytical case of a sinusoidal signal [eq. (12)] shows that $F(\omega) \neq 0$ only at the specific angular frequencies $+\omega_{0}$ and $-\omega_{0}$. The Fourier Transform of a pulsed signal, on the other hand, with a given width and periodicity leads to to $F$ $(\omega) \neq 0$ for, $k \cdot \omega_{0}, k \in \mathbb{N}$ (multiples of $\omega_{0}$ ). ${ }^{18}$ Only a cosine or sine wave results in a single peak at a specific frequency. The sharkskin structure (Figure 2) in the time domain is more likely to correspond to a pulse signal, especially after some image enhancement (section 4) where the pixel brightness is set to 0 (valley of a groove of the instability) or 255 (peak of a groove of the instability). Therefore, care must be taken if in the frequency domain peaks appear at multiples of the main frequency as it might be a mathematical artifact. The following discussion focuses only on the main modulation of the extrudates' surfaces. Hence, only the signal at the frequency with the highest magnitude (in the frequency domain) needs to be considered. For the Fourier transform employed on the time dependent pressure data please refer to the following publications, where the method is described in detail. ${ }^{4,5,15-17}$

\section{Fourier Analysis of an Image}

To apply the Fourier Transform simply selecting one row of pixels covering the whole $x$ direction might be the easiest but not the most accurate solution, as it is likely to miss or overrate some information as the analysis is based on experimental data. Therefore, the part of the picture showing the instability is split in four different regions (Figure 3 ). This allows averaging in $y$ direction. Regions $1-3$ have a height of around 100 pixels in $y$ spanning the entire $x$ direction. Region 4 however captures the whole instability from top to bottom. For each region, the brightness of all the pixels in $y$ direction is averaged for one specific coordinate in $x$ direction. This leads to an averaged brightness for each pixel in $x$ direction. Some samples might even show ripples on the side of the extrudate. This was not the case for our samples, but if they appear, an additional region including them should be considered as they can be detected using the sharkskin option from Göttfert and will lead to and additional peak in the FT spectrum.

The averaged pixel brightness in the time domain is displayed in Figure 4(a). As can be seen the peaks of all the different regions overlap in most of the cases. Therefore, for this example it should not matter which region is chosen. As explained in section 3 the conversion to the time domain is done under assumption of laminar and not plug flow, although this will occur at the die exit.

Figure 4(b) shows the result of the FFT. A major peak appears between $5 \mathrm{~Hz}$ and $6 \mathrm{~Hz}$ for each region. This is the characteristic frequency of the sharkskin instability at $\dot{\gamma}=33 \mathrm{~s}^{-1}$. As expected from the Time Domain data (Figure $4 \mathrm{a}$ ) the characteristic frequency is basically the same for all the different regions. Furthermore, a second peak of much weaker amplitude seems to appear between $10 \mathrm{~Hz}$ and $12 \mathrm{~Hz}$. This second peak is due to the small light reflections in between the main grooves. As the image is not completely dark in between the flow instability this will lead to additional shorter peaks in the time domain, resulting in the end in a less pronounced peak at a higher frequency in the FT graph. To improve the results, some numerical techniques to enhance the quality of the output are employed. The different methods are explained in the following section.

\section{IMAGE QUALITY ENHANCEMENT}

A typical image with a well-developed sharkskin instability is shown in Figure 5. The picture has been taken with a standard

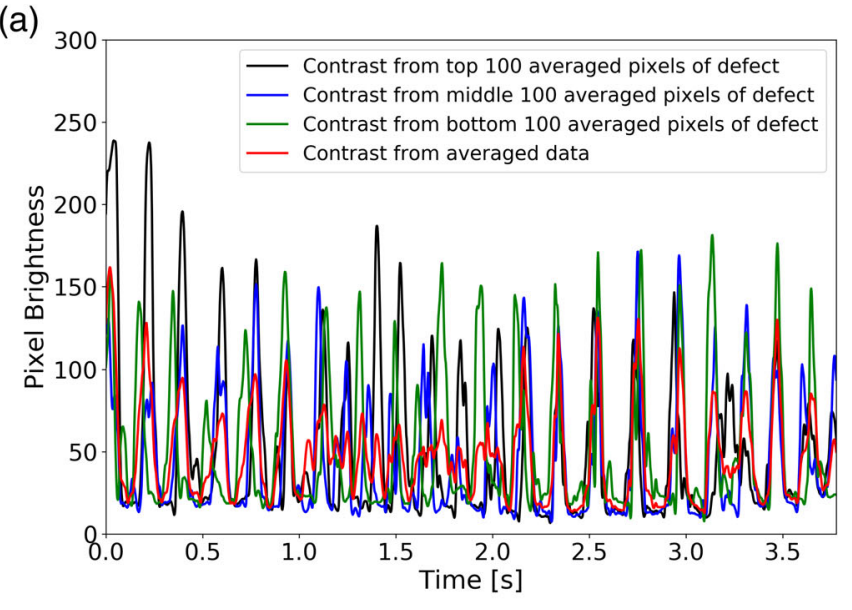

(b)

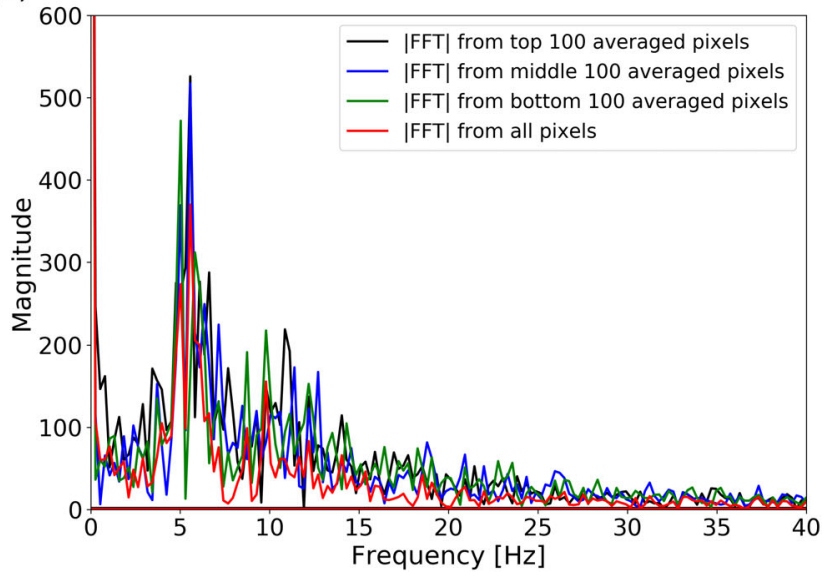

Figure 4. (a) Time domain signal representing averaged brightness of the image for different regions from Figure 3, (b) FFT of time domain signal from Figure 3. [Color figure can be viewed at wileyonlinelibrary.com] 


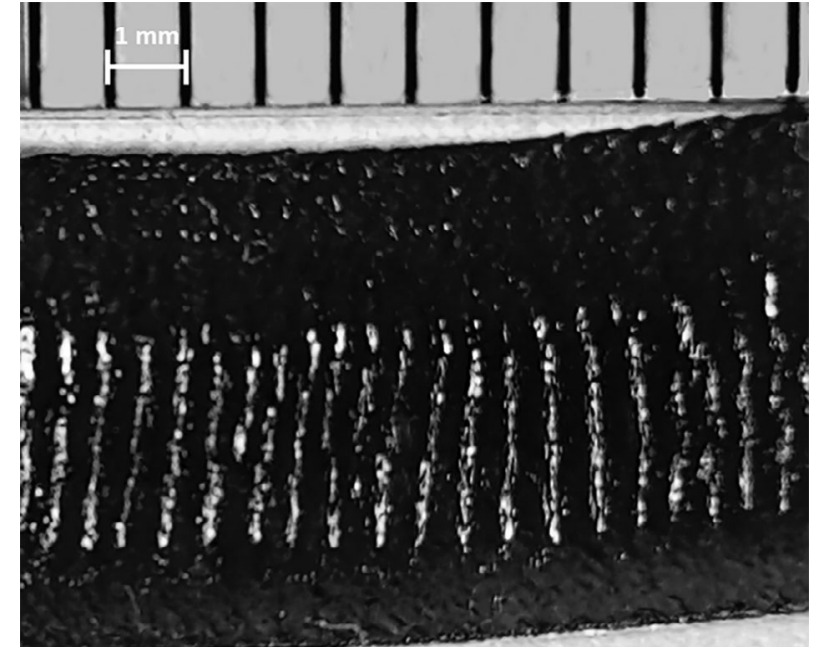

Figure 5. Sharkskin instability on unfilled SBR extruded at a shear rate of $\dot{\gamma}=33 \mathrm{~s}^{-1}$.

camera under optimum lighting conditions. For all the following pictures the distance between two lines corresponds to $1 \mathrm{~mm}$, and all the presented extrudates in the pictures have a total length of $1 \mathrm{~cm}$.

The next step consists in improving the picture with respect to the instability. The OpenCV (Open Source Computer Vision Library $)^{19}$ for python offers many functions to enhance the quality of an image. First, the image is imported in gray scale and the contrast and brightness are adapted. A picture is a $2 D$ array with a given number of pixels in $x$ and $y$ direction. As the image is gray scale, it only has one value associated to each pixel, corresponding to the brightness with values ranging from 0 (black) to 255 (white). Increasing/decreasing the contrast means multiplying/dividing all the pixels by a given value $\alpha$ [eq. (13)]. Increasing/decreasing the brightness corresponds to adding/subtracting a value $\beta$ to all the pixels [eq. (13)]. Mathematically this can be formulated for a specific pixels located at the $(x, y)$ coordinate $(i, j)$ as

$$
g(i, j)=\alpha f(i, j)+\beta
$$

where $f(i, j)$ is the original (source) image pixel, $g(i, j)$ the output image pixel, $\alpha>0$ controlling the contrast and $\beta$ the brightness. $\alpha$ and $\beta$ might also be referred to as gain parameters. Instead of looping through all the pixels in $x$ and $y$ direction and instead of applying eq. (13), using OpenCVs function "convertScaleAbs" is applied directly, allowing more efficient changes of the brightness and contrast with respect to computational time.

Figure 6 compares the original [Figure 6(a)] with the contrast and brightness enhanced picture [Figure 6(b)]. The sharkskin instability can now even better be distinguished from the background. For an uniformly illuminated sample, adapting the contrast and brightness is already sufficient to improve the results.

Figure 7(a) shows a nonuniformly illuminated picture of an SBR sample filled with $112 \mathrm{phr}$ of silica. In contrast to Figure 6(a), Figure $7(\mathrm{a})$ is much darker on the left as on the right. Changing the brightness and contrast would not lead to good results as typically the image becomes overexposed on one side (right) and even darker on the other side (left) [Figure 7(b)]. One possibility might be to use a binary threshold. In this case, all the pixels with a brightness below the threshold brightness will be set to a given value and all the pixels above the threshold will be set to another brightness. Therefore a threshold for the pixel brightness is defined $(i, j)_{\text {thresh }} \in[0,255]$, and $g(i, j)$ corresponds again to the output.

$$
g(i, j)=\left\{\begin{array}{l}
0 f(i, j)<f(i, j)_{\text {thresh }} \\
255 f(i, j)>f(i, j)_{\text {thresh }}
\end{array}\right.
$$

If the brightness of the actual pixel $f(i, j)$ is below the threshold $f(i, j)_{\text {thresh }}$ the brightness is set to 0 or to another predefined value. Otherwise it is set to 255 or another predefined value.

Figure 8(a) shows the effect of a binary threshold $f(i, j)_{\text {thresh }}=80$. As can be seen on the right, the picture is overexposed whereas on the left we observe slightly more structure as before. If the threshold is increased to $f(i, j)_{\text {thresh }}=150$ as shown in Figure 8 (b) the over saturation on the right side of the picture is eliminated but nearly all the information on the left side is lost as it is
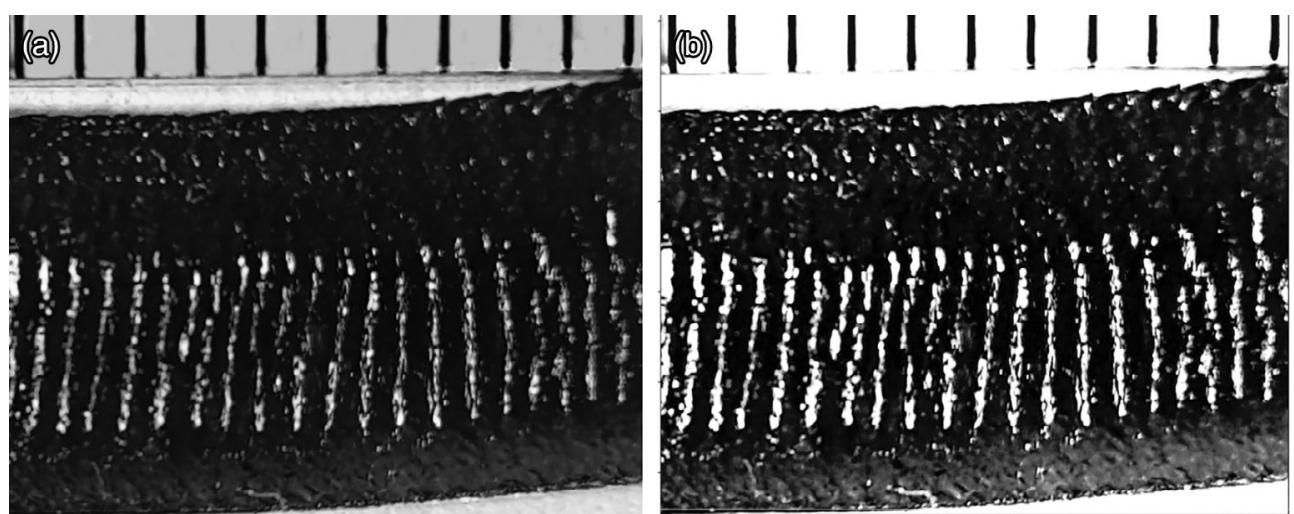

Figure 6. Unfilled SBR extrudate, extruded at a shear rate of $\dot{\gamma}=33 \mathrm{~s}^{-1}$. (a) Original image (b) contrast and brightness enhanced image with $\alpha=2, \beta=-30$. 

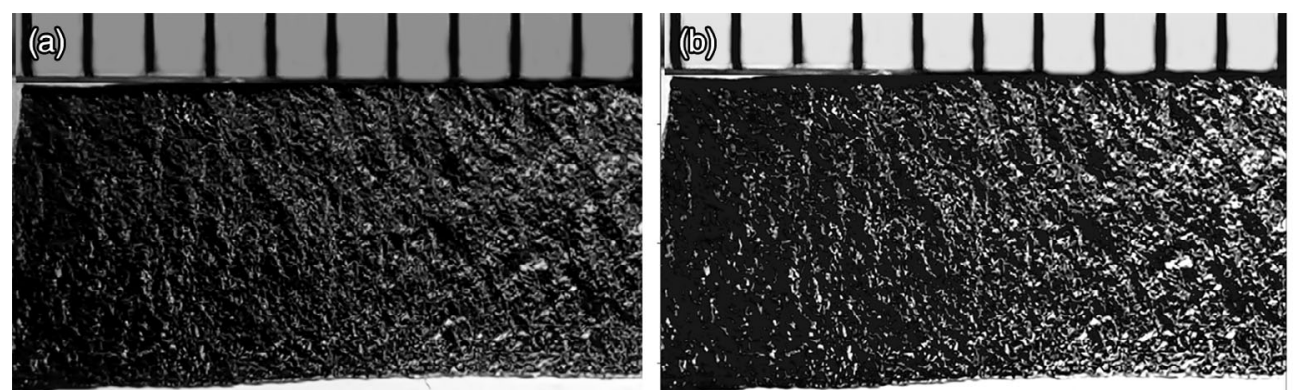

Figure 7. SBR+112 phr silica extrudate, extruded at a shear rate of $\dot{\gamma}=200 \mathrm{~s}^{-1}$ (a) original picture (b) contrast and brightness enhanced image with $\alpha=1.5, \beta=-30$.

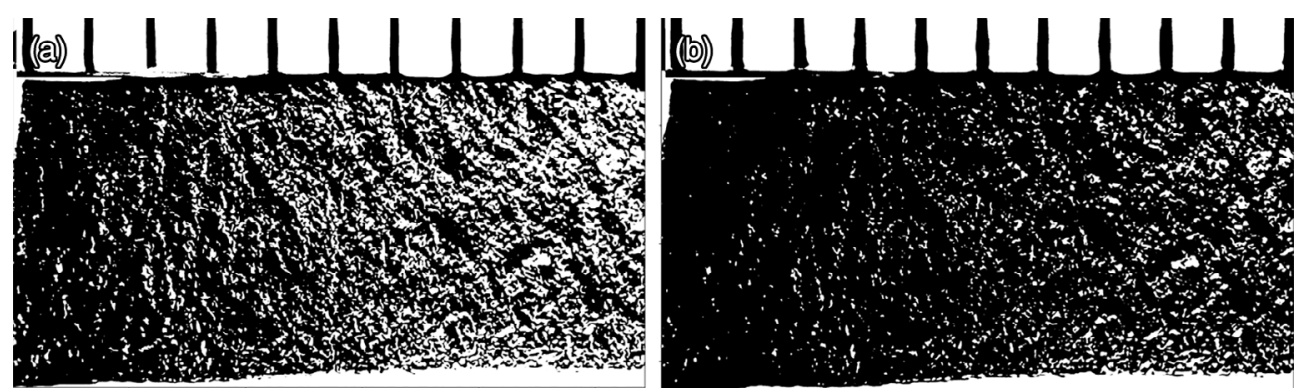

Figure 8. Binary threshold filter applied to SBR+112 phr silica extrudate, extruded at a shear rate of $\dot{\gamma}=200 \mathrm{~s}^{-1}$ with $(\mathrm{a}) f(i, j)_{\text {thresh }}=80,(\mathrm{~b}) f(i, j)_{\text {thresh }}=150$.

getting darker and darker. Until now, the OpenCV function "threshold" with "THRESH_BINARY" has been used. A better solution for images with different lightning conditions in different areas is the use of adaptive threshold filters. In this case, the algorithm calculates the threshold for a small, user defined, region. This leads to different threshold for different regions and therefore to better results in the case of nonuniform illumination. OpenCv has for example two adaptive filters. One uses the mean of the neighborhood area as threshold value. The second one employs a threshold value which is the weighted sum of neighborhood values where weights are Gaussian windows. The Gaussian filter is employed in the following analysis as it lead to better results for these specific samples. In OpenCV the function "adaptiveTreshold" is used with "ADAPTIVE_THRESH_ GAUSSIAN_C”. The size of the neighborhood area is referred to as "blocksize" and it is even possible to add or subtract a given value "const" to or from the image respectively, acting similar as the $\beta$ parameter controlling the brightness.
Applying the Gaussian filter to the original picture [Figure 9(a)] leads to Figure 9(b). This filter recovers most of the structure which could not have been recovered by simple changing the brightness and contrast. Furthermore, this technique makes it unnecessary in some cases to adjust the brightness and contrast beforehand.

Care must however be taken for the size of the neighborhood area. If chosen too small it might reveal nonphysical features [Figure 10(a)], if chosen too big it might hide them [Figure 10(b)]. The results show that it is generally better to choose the neighborhood area too big rather than too small.

\section{RESULTS}

\section{Slit Die}

The following measurements have been carried out with the slit die with dimensions $H=0.5 \mathrm{~mm}, W=5 \mathrm{~mm}$ with 3 piezoelectric pressure transducers which are located along a slit die. Figures 11 (a)-15(b) show the samples that are going to be analyzed in the
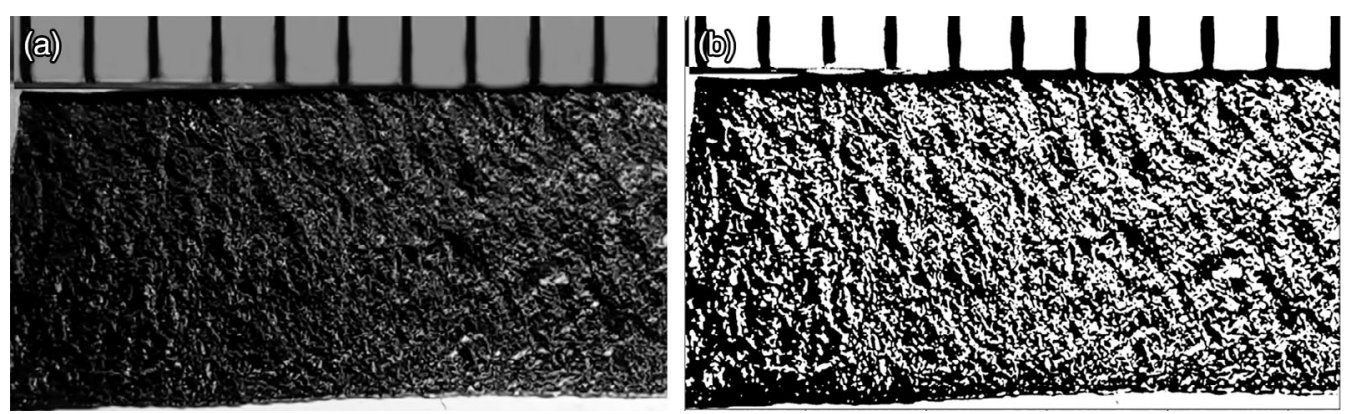

Figure 9. SBR+112 phr silica, extruded at a shear rate of $\dot{\gamma}=200 \mathrm{~s}^{-1}$. (a) Original image (b) Enhanced image using Gaussian filter with blocksize $=501$, $\alpha=1, \beta=0$. 

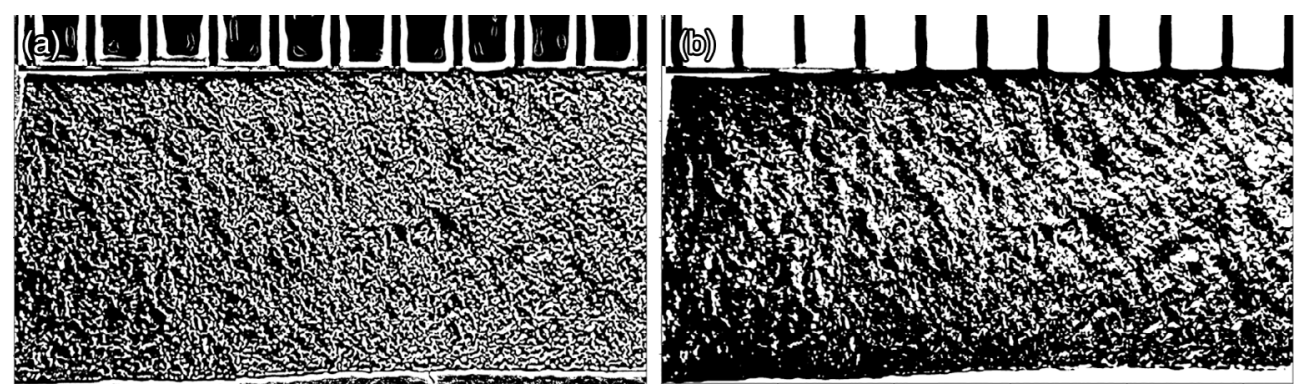

Figure 10. SBR+112 phr silica, extruded at a shear rate of $\dot{\gamma}=200 \mathrm{~s}^{-1}$ (a) Gaussian filter with blocksize $=51$, (b) Gaussian filter with blocksize $=1501$.
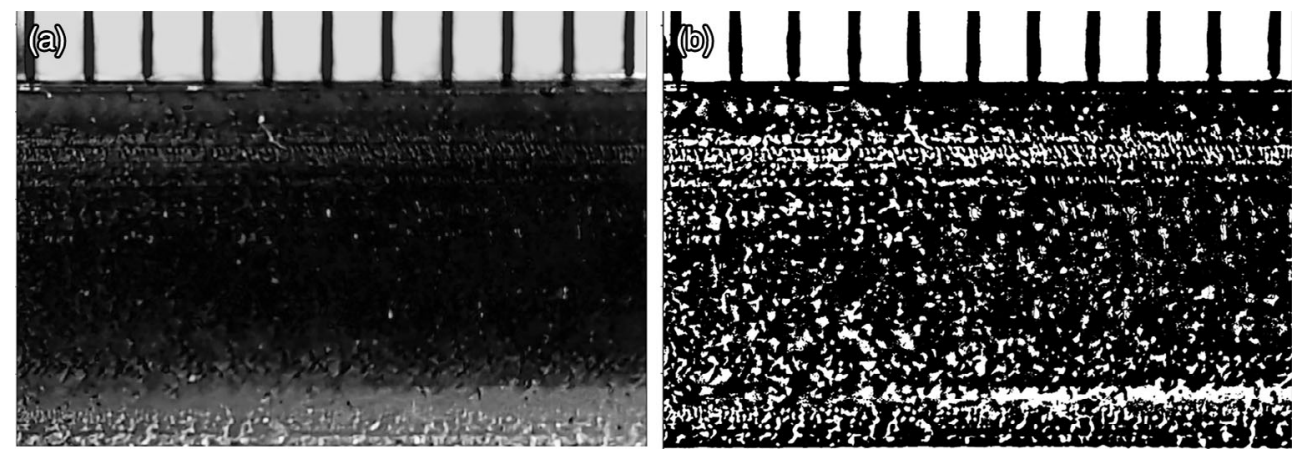

Figure 11. Unfilled SBR extruded at a shear rate of $\dot{\gamma}=10 \mathrm{~s}^{-1}$ (a) $\alpha=1.2, \beta=-50$, (b) Gaussian filter with blocksize $=301$, const $=-5$.

following graphs. For each set of two figures, Figure 11(a) corresponds to the original picture with enhanced contrast and brightness and Figure 11(b) after applying the Gaussian filter to picture (a). The shear rates, amount of silica and parameters used for improving the image quality are indicated below the figures.

In Figures 11(a)-13(b) for the shear rates $\dot{\gamma}=33 \mathrm{~s}^{-1}, 60 \mathrm{~s}^{-1}$ the periodic instability (sharkskin) can clearly be seen. After applying the Gaussian filter, the defect appears even more apparent. In Figure 11(a) barely any effect is visible, after a close look and especially after applying the gauss filter, the onset of sharkskin on the top of the image becomes clearer [Figure 11(b)]. Applying the filter in Figure 11(b) leads to some bright spots, but as they are not periodic they should not influence the FFT too much. In this case, it might even be better not to use the Gaussian filter but only to improve the brightness and contrast. Investigating Figure 13(b) in detail, one might question the validity of the classification criterion "Sharkskin manifests as periodic structure with an amplitude of a few tens to hundreds of microns over the whole extruded sample surface" given in the introduction as the amplitude becomes significantly higher as a few microns. It might be better to define sharkskin more general as "continuous surface instability". This would exclude gross melt fracture as it is a volume distortion where the whole sample is deformed. The unfilled SBR sample however, only shows the periodic pattern on one side of the extrudate, therefore it is still a surface instability. Furthermore, using "continuous" in the definition excludes stick slip, as this consists of alternating smooth and rough regions.

Figure 14(a) shows the contrast and brightness enhanced SBR sample with $30 \mathrm{phr}$ of silica. After applying the Gaussian filter, the whole surface of the sample can clearly be identified.
Figure 15(a) shows the contrast and brightness enhanced SBR sample with $112 \mathrm{phr}$ of silica. In contrast to Figure 14(a), this sample is nonuniformly illuminated, as it is much darker on the left compared to the right. This is the main reason why the Gaussian filter is used. In Figure 15(b) apparently most of the surface could be recovered with help of the filter. For the images represented in Figures 11(a)-15(b) the FFT from the pressure data is compared to the optical analysis method (e.g., Figure 16). Only for the sample represented in Figure 12 (a) the pressure and enhanced brightness data are shown which are used to compute the FFT to illustrate how it looks in the ideal case (see Figure 17). The FFT deduced from the piezoelectric pressure transducers (for example Figure 16(b) has been normalized by the mean pressure of the corresponding pressure transducer. As the FFTs lines overlap due to normalization at a value around 1, they have been shifted vertically to improve the readability of the results. The FFT from the piezoelectric pressure transducer $P 1$ is always shifted up and the FFT from the piezoelectric pressure transducer $P 3$ is always shifted down with respect to $P 2$ which is not shifted. Hence some FFT graphs show a negative value for $P 3$. As mentioned in section 2 the piezoelectric pressure transducers have always recorded the time domain signal for $20 \mathrm{~s}$.

The start of the onset of sharkskin might be seen at the top in Figure 11(a) but there is no characteristic peak at any specific frequency in the FFT of the pressure data [Figure 16(b)]. The optical analysis shows different peaks for different regions. It seems that in some regions the bright spots resulting from the Gaussian filter seem to be more or less periodic [Figure 16(a) blue, red curves], but there is no clear main peak overlapping for all the 

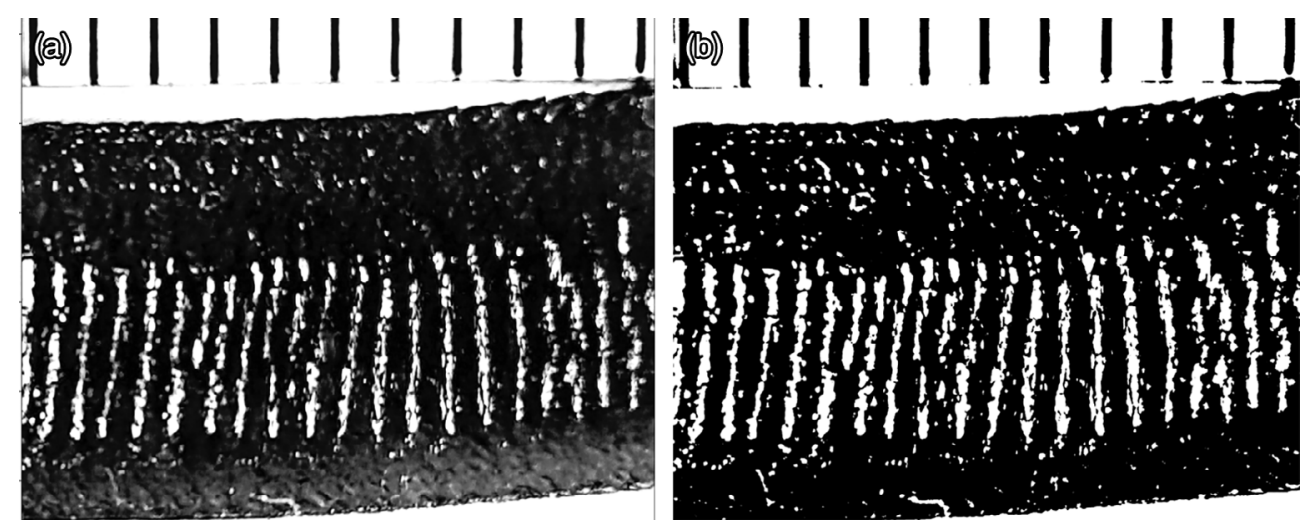

Figure 12. Unfilled SBR extruded at a shear rate of $\dot{\gamma}=33 \mathrm{~s}^{-1}$ (a) $\alpha=2, \beta=-30$, (b) Gaussian filter with blocksize $=1001$, const $=-10$.
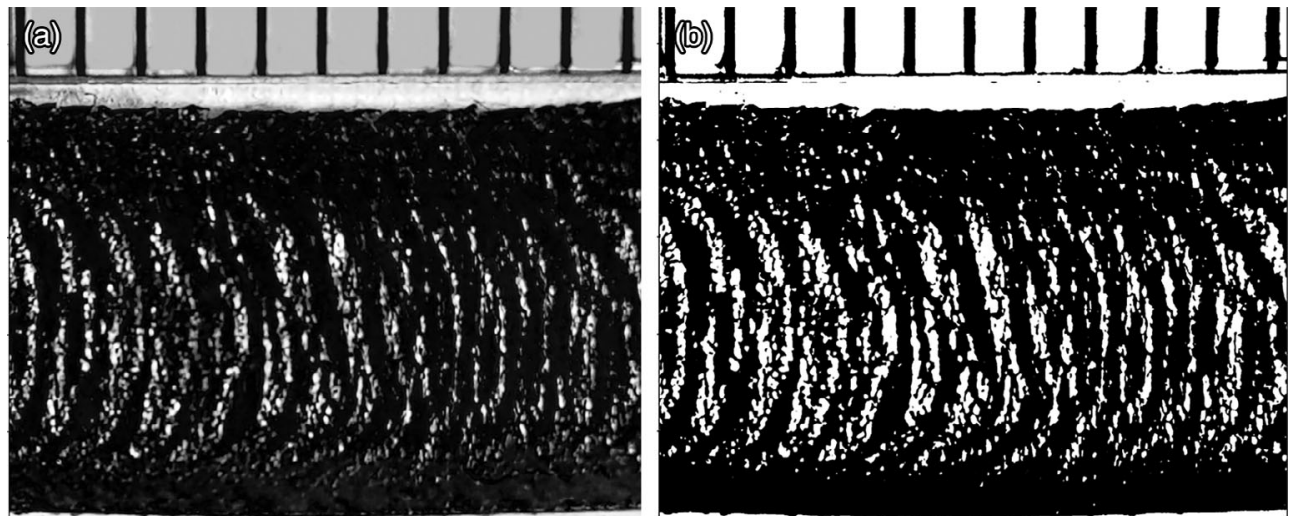

Figure 13. Unfilled SBR extruded at a shear rate of $\dot{\gamma}=60 \mathrm{~s}^{-1}$ (a) $\alpha=1.2, \beta=-30$, (b) Gaussian filter with blocksize $=1001$, const $=-2$.
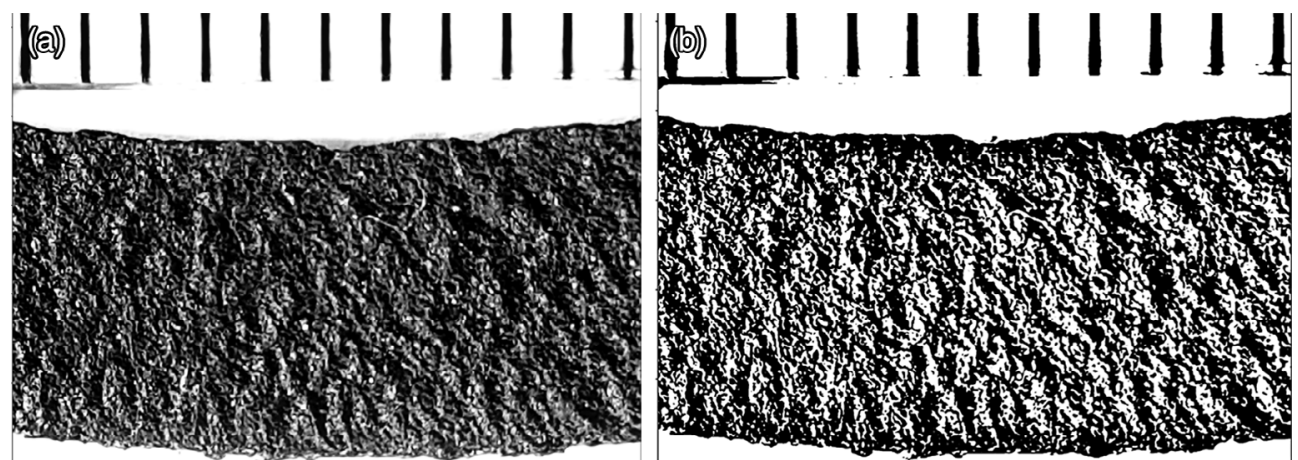

Figure 14. SBR with $30 \mathrm{phr}$ silica extruded at a shear rate of $\dot{\gamma}=10 \mathrm{~s}^{-1}$ (a) $\alpha=1.5, \beta=0$, (b) Gaussian filter with blocksize $=501$, const $=0$.
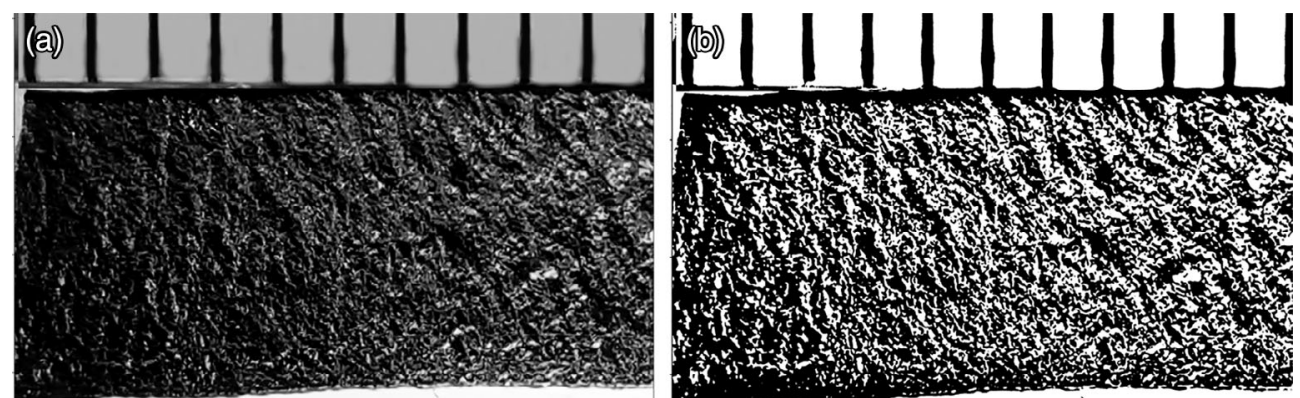

Figure 15. SBR with $112 \mathrm{phr}$ silica at $\dot{\gamma}=200 \mathrm{~s}^{-1}$ (a) $\alpha=1.0, \beta=0$, (b) Gaussian filter with blocksize $=501$, const $=0$. 
(a)

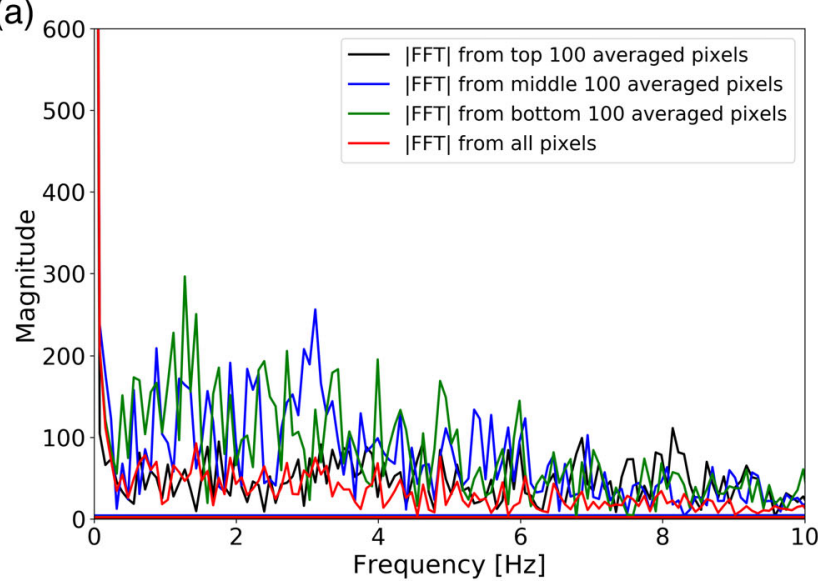

(b)

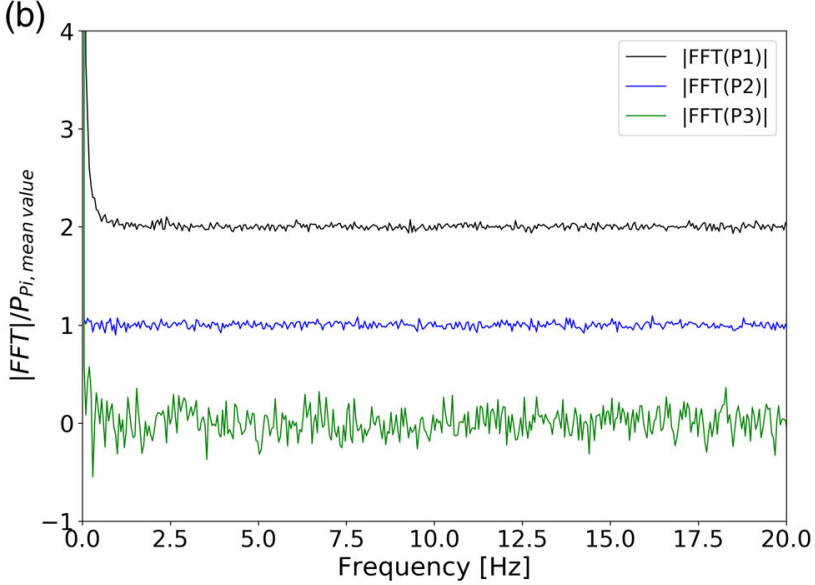

Figure 16. Unfilled SBR at $\dot{\gamma}=10 \mathrm{~s}^{-1}$ (a) FFT result from optical analysis, (b) normalised and for readability vertically shifted FFT result from piezoelectric pressure transducers. [Color figure can be viewed at wileyonlinelibrary.com]

regions. Especially, the red curve, which averages over the whole sample does not show any characteristic peak.

In Figure 12(a,b), a very clear periodic pattern can be observed. This remains after converting the picture into brightness versus time plot for different regions [Figure 17(a)]. For all the four regions the peaks in the time domain are mostly overlapping. The corresponding Fourier transform is represented in Figure 17(b). A very pronounced characteristic peak appears at a frequency of around $5 \mathrm{~Hz}$. Figure 17(c) shows the normalized pressure data vertically shifted with respect to each other. Although we measured for $20 \mathrm{~s}$ we only display one single second to highlight the pressure oscillations. Three oscillations per second can be counted, therefore expecting a peak at a characteristic frequency of $3 \mathrm{~Hz}$ which is confirmed in the relative FFT [Figure 17(d)]. This shows that the characteristic frequencies obtained from the pressure data differ from the ones obtained by the image analysis, but still are the same order of magnitude. One of the reasons for the difference is explained in section 6 . As stated by Wilhelm et al. ${ }^{5,15-17}$ The pressure oscillation can be measured throughout the whole die although the origin of sharkskin is usually expected to be mainly linked to the die exit. ${ }^{1,2}$ Another interesting observation is that the pressure fluctuations of the piezoelectric pressure transducer $P 1$ of the unfilled SBR sample extruded at $\dot{\gamma}=33 \mathrm{~s}^{-1}$ shows a stronger response as $P 2$ and $P 3[-$ Figure 17(c)]. This is surprising because in the publication ${ }^{17}$ it is reported that for the sharkskin instability the relative pressure fluctuations should be strongest at the die exit (piezoeletric pressure transducer $P 3$ ), supporting the theory of sharkskin as a die exit effect. These data were recorded for an ethylene/1-octene copolymer from Dow with a short chain branching (SCB) incorporation of $7 \mathrm{~mol} \%$. Our data however show that for SBR the relatively strongest and clearest signal is measured in $P 1$, closest to the barrel. In contrast to the polyethylene sample it seems that the origin of this instability is inside the barrel.

The analysis of unfilled SBR at a shear rate of $\dot{\gamma}=60 \mathrm{~s}^{-1}$ with a very pronounced sharkskin [Figure 13(a,b)] is challenging as it is not clear from the picture where one peak ends and another one starts. Furthermore, in contrast to unfilled SBR extrudate at a shear rate of $\dot{\gamma}=33 \mathrm{~s}^{-1}$ the peaks are not straight anymore but have a slightly parabolic shape. The FFT of the top and bottom region [Figure 18(a), black and green curves] have both characteristic peaks around $5 \mathrm{~Hz}$ whereas the middle region [Figure 18 (a), blue curve] has a peak around $15 \mathrm{~Hz}$. This difference becomes obvious by examing Figure 13(b) more closely. The peaks of the instability linked to the top and bottom region can be nicely distinguished from each other as the width of the instability is narrower. In the middle of the sample, however as mentioned before it is not clear where a peak ends and another one starts. Therefore it seems that one single peak in the instability is actually counted as two peaks. If the average however is carried out over the whole defect [Figure 18(a), red curve] the characteristic frequency is identical to the top and bottom region. The relative FFT from the pressure data [Figure 18(b)] also shows a clear peak at a frequency of $4 \mathrm{~Hz}$. Which is close to the result obtained by the optical analysis.

Figure 19(a,b) shows the FFT of the optical pressure data from the SBR with $30 \mathrm{phr}$ silica [Figure 14(a,b)]. From the picture, no clear wavelength of the instability can be seen. This is also reflected from the optical and pressure FFT where no peak can be observed. Only damped oscillations from low to higher frequencies occur.

Finally, the SBR compound with $112 \mathrm{phr}$ silica is analysed. As for the $30 \mathrm{phr}$ sample, no periodic pattern can be observed in Figure 15(a,b). This is again reflected in the FFT of the image [Figure 20(a)] and pressure data [Figure 20(b)]. It should however be noted that the FFT from the image analysis shows a much lower resolution compared to the pressure data. This due to the fact that the resolution of FFT from the optical analysis is linked of the size of a pixel. To improve the result, a camera with a higher resolution is required. As can be seen in Figure 20(b) at 5, $12,16 \mathrm{~Hz}$ there seem to be peaks hidden in the noisy data. Using multiples of the standard deviation of the FFT in the frequency range $30-40 \mathrm{~Hz}$, the noise can be removed from the data, resulting in a smoother graph as shown in Figure 21. However, these oscillations only seem to be present in the $P 1$ piezoelectric pressure transducer closest to the barrel

\section{Round Hole Capillary}

Finally, the optical analysis is tested on the Rheograph 25 equipped with a round hole capillary with a length to diameter 
(a)

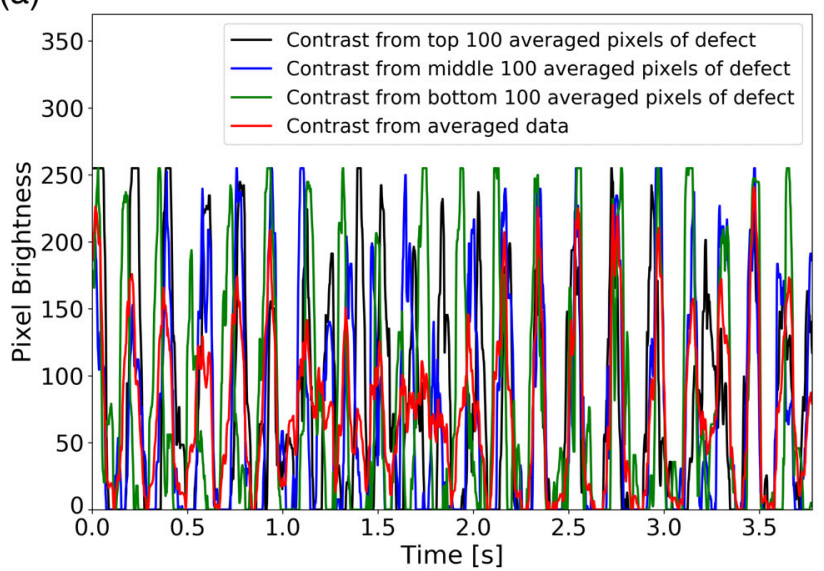

(c)

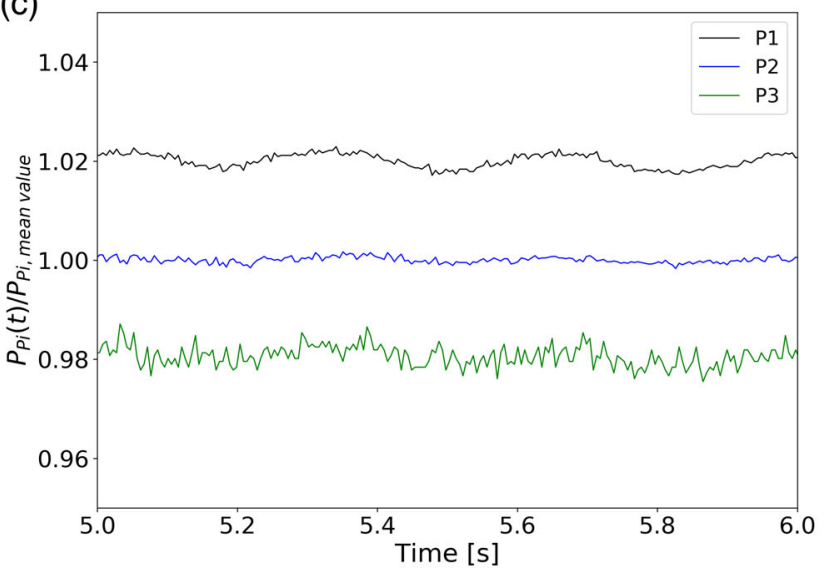

(b)

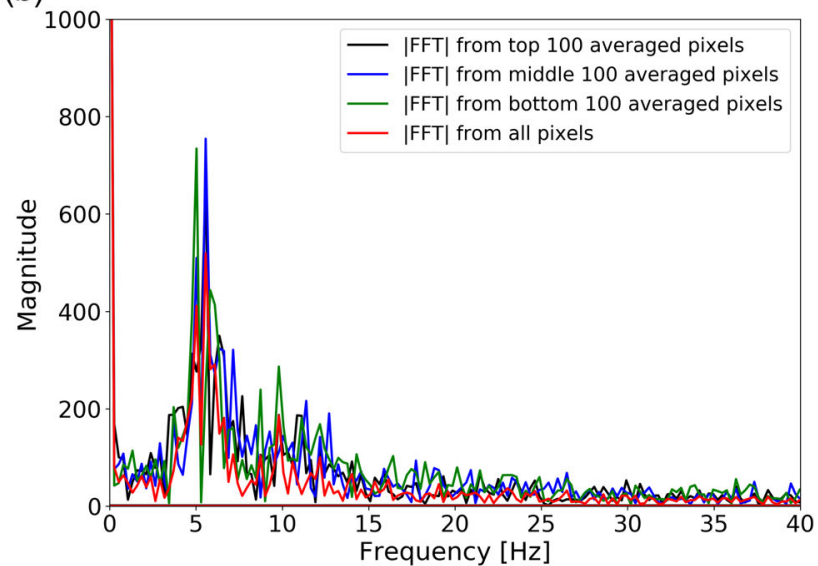

(d)

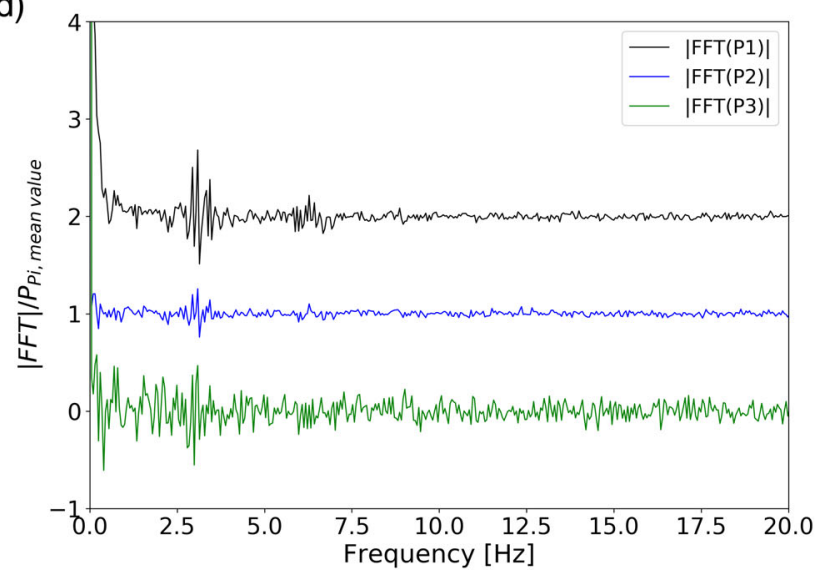

Figure 17. Unfilled SBR extruded at a shear rate of $\dot{\gamma}=33 \mathrm{~s}^{-1}$ (a) Time domain signal representing averaged brightness of the image for different regions, (b) FFT result from optical analysis, (c) $1 \mathrm{~s}$ window from $20 \mathrm{~s}$ normalized and for readability vertically shifted time domain signal from piezoelectric pressure transducers, (d) normalised and for readability vertically shifted FFT result from piezoelectric pressure transducers. [Color figure can be viewed at wileyonlinelibrary.com]

ratio in $\mathrm{mm}$ of $L / D=30 / 3$. The unfilled $\mathrm{SBR}$ is used as it develops a very pronounced sharkskin instability.

The contrast and brightness enhanced picture of the SBR sample extruded at $\dot{\gamma}=10 \mathrm{~s}^{-1}$ through the round hole capillary is represented in Figure 22(a). After applying the Gauss filter the sharkskin defect becomes much more pronounced [Figure 22(b)].

From the time domain graph [Figure 23(a)] by applying the FFT the frequency domain graph [Figure 23(b)] with several peaks (a)

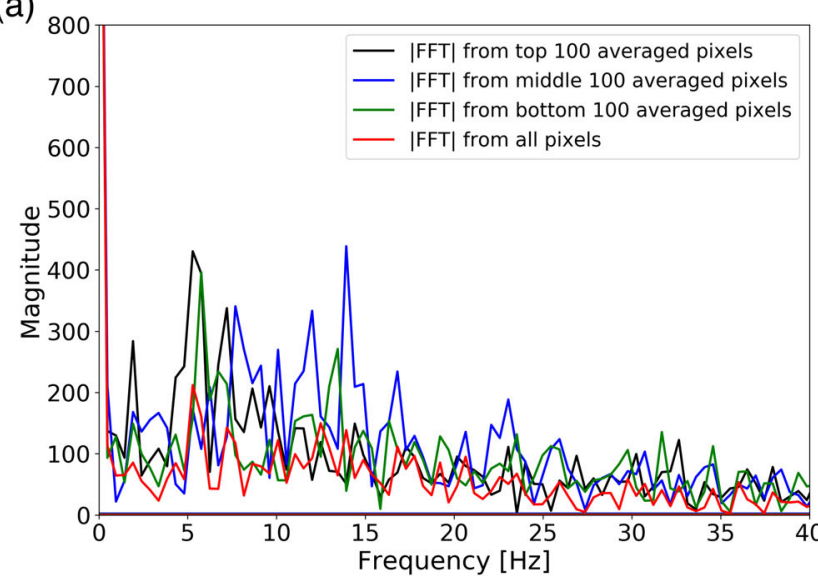

(b)

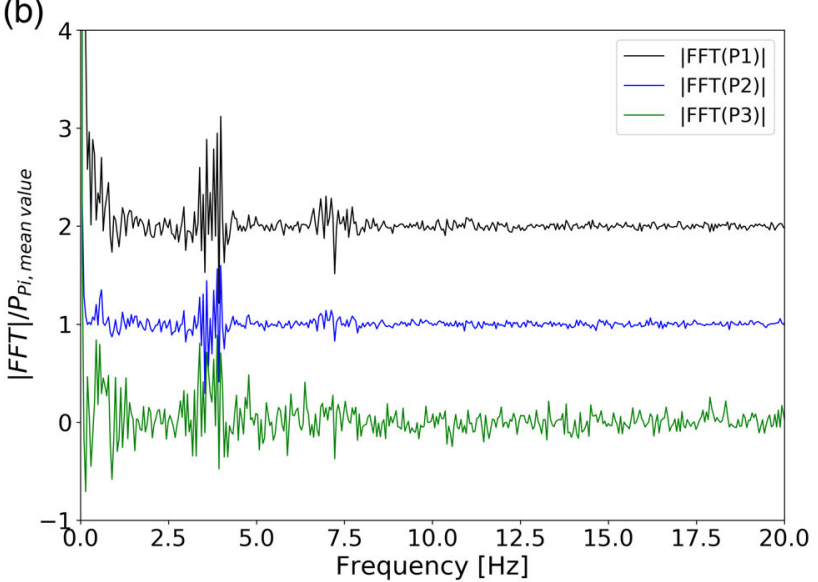

Figure 18. Unfilled SBR at $\dot{\gamma}=60 \mathrm{~s}^{-1}$, (a) FFT result from optical analysis, (b) normalized and for readability vertically shifted FFT result from piezoelectric pressure transducers. [Color figure can be viewed at wileyonlinelibrary.com] 
(a)

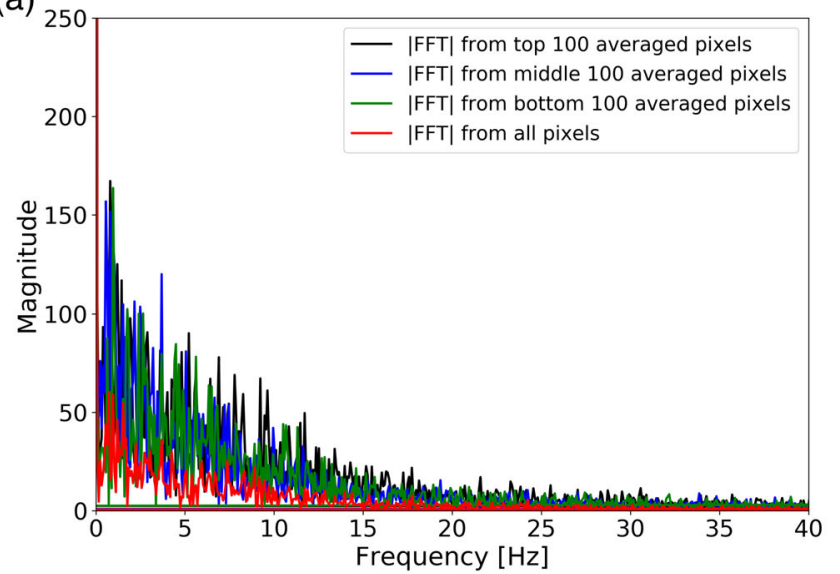

(b)

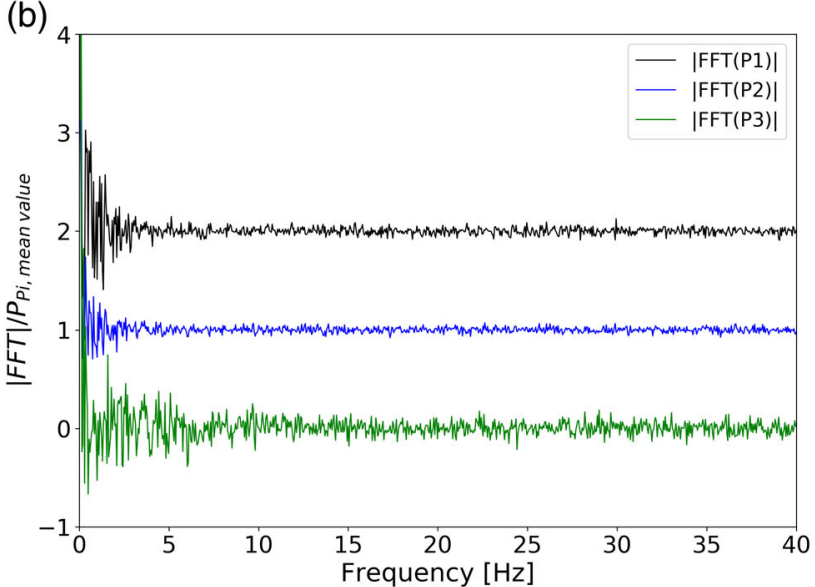

Figure 19. SBR $+30 \mathrm{phr}$ silica at $\dot{\gamma}=10 \mathrm{~s}^{-1}$, (a) FFT result from optical analysis, (b) normalized and for readability vertically shifted FFT result from piezoelectric pressure transducers. [Color figure can be viewed at wileyonlinelibrary.com]

(a)

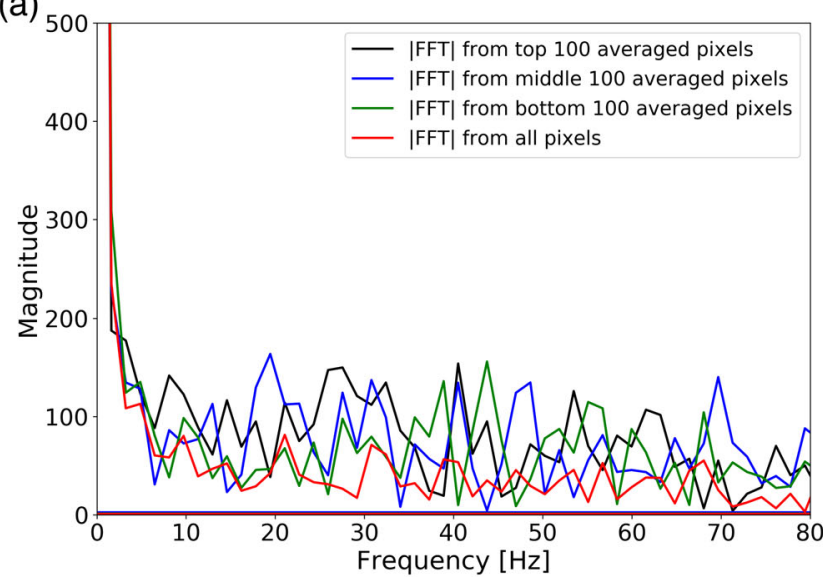

(b)

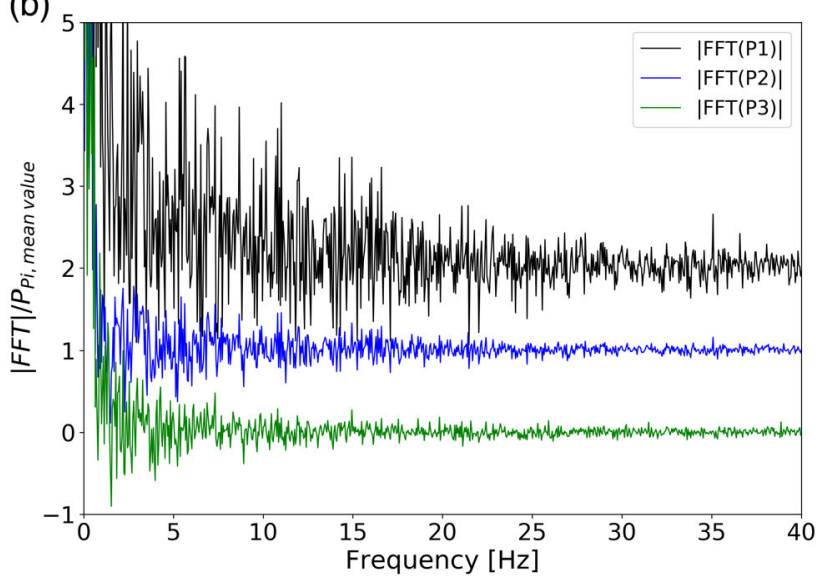

Figure 20. SBR $+112 \mathrm{phr}$ silica at $\dot{\gamma}=200 \mathrm{~s}^{-1}$, (a) FFT result from optical analysis, (b) normalised and for readability vertically shifted FFT result from piezoelectric pressure transducers. [Color figure can be viewed at wileyonlinelibrary.com]

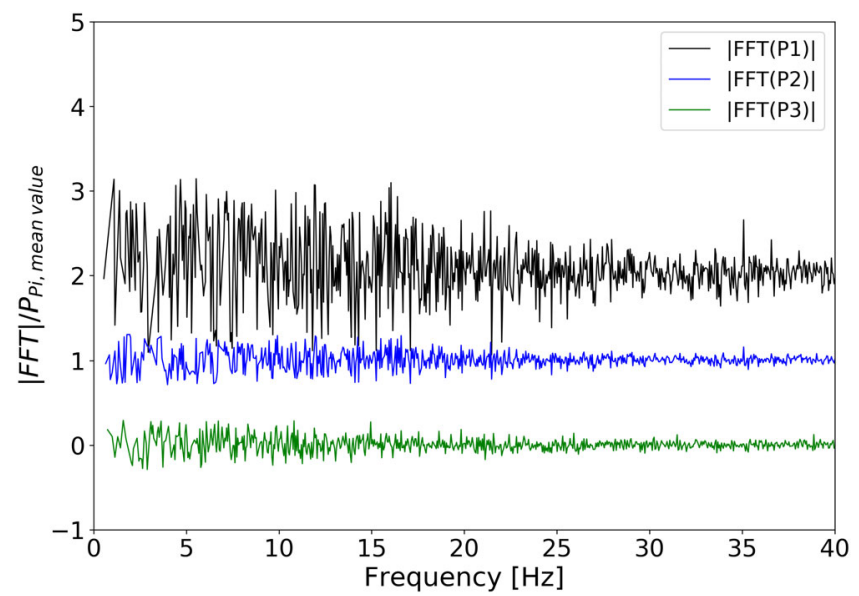

Figure 21. SBR $+112 \mathrm{phr}$ silica at $\dot{\gamma}=200 \mathrm{~s}^{-1}$, (a) FFT result from optical analysis, (b) by standard deviation corrected, normalised and for readability vertically shifted FFT result from piezoelectric pressure transducers. [Color figure can be viewed at wileyonlinelibrary.com] ranging between $7-10 \mathrm{~Hz}$ for the different regions is obtained. Examining Figure 22(b) this is not surprising as the grooves of the sharkskin defect are not perfectly perpendicular to the extrusion direction. Furthermore, it appears that a single groove may split into two grooves or merge into one, explaining why different characteristic peaks are obtained depending on the region. Therefore, it is reasonable to consider the FFT of the whole defect (red line) as this automatically averages over the grooves.

\section{CHARACTERISTIC FREQUENCY PEAK SHIFT}

Palza at al. ${ }^{20}$ reported a difference in the frequency of the surface instability measured from the piezoelectric pressure transducers and the extrudate. They measured the characteristic length of the instability directly from the extrudate, defined as the distance between two consecutive ridges in the processed sample in the solid state, and the average velocity of the melt extrudate. For a polyethylene sample displaying sharkskin, they measured a characteristic frequency of $22 \mathrm{~Hz}$ with the piezoelectric pressure transducers but obtained a characteristic frequency of $60 \mathrm{~Hz}$ from 


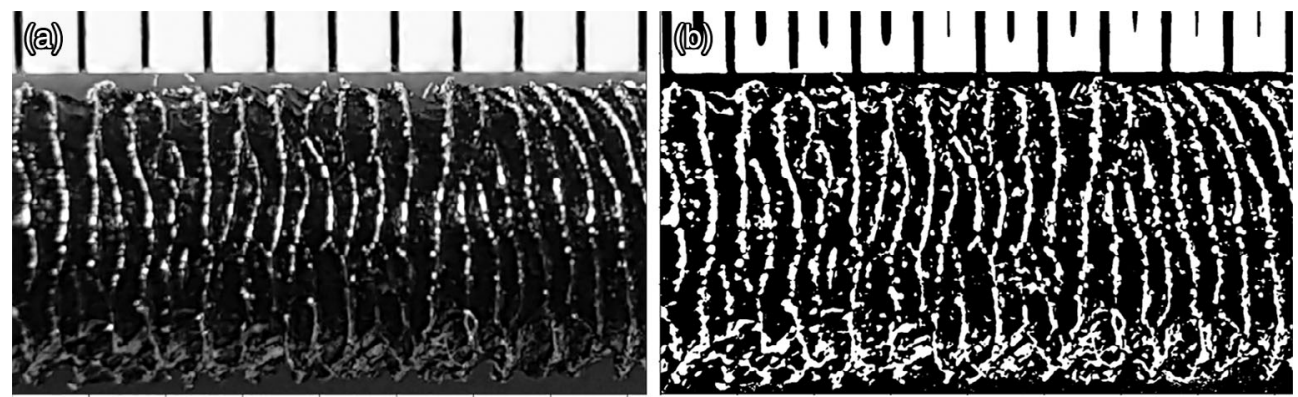

Figure 22. SBR extruded at a shear rate of $\dot{\gamma}=10 \mathrm{~s}^{-1}$ (a) $\alpha=1.2, \beta=0$ (b) Gaussian filter with blocksize $=201$, const $=-5$.

the processed extrudate. They assume this difference occurs due to the change from lamellar flow inside the die to a plug flow outside, die swell phenomena, uncertainties linked to the estimation of the extrudate velocity, and so forth. Again, a shift between the pressure related characteristic frequency and the characteristic frequency from the optical analysis is observed. To investigate this in more detail a second slit die with only one single piezoelectric pressure transducer is used. The measurements are carried out at a shear rate of $\dot{\gamma}=33 \mathrm{~s}^{-1}$. With dimensions $H=0.3 \mathrm{~mm}, W=3 \mathrm{~mm}$ this leads to an extrusion speed of $1.65 \mathrm{~mm} \mathrm{~s}^{-1}$ [eq. (6)]

FFT from piezoelectric pressure transducer $P 2$ with a characteristic frequency at $1.7 \mathrm{~Hz}$.

The highest peak corresponding to the characteristic frequency measured from the piezoelectric pressure transducer [Figure 24(b)] is located at $1.7 \mathrm{~Hz}$. The optical analysis [Figure 25(b)] however gives a characteristic peak at $4 \mathrm{~Hz}$. The shrinking of the extrudate after the extrusion is suspected to be the main reason for this difference. To correct for this, instead of taking the velocity from the shear rate directly, the extrusion time $t_{\text {ext }}=500 \mathrm{~s}$ is recorded and the length of the extrudate after the extrusion, corresponding to $l_{\text {ext }}=495 \mathrm{~mm}$ is used. This leads to an "apparent" velocity of

$$
v_{a p p}=\frac{l_{\text {ext }}}{t_{\text {ext }}} \approx 1 \mathrm{~mm} \mathrm{~s}^{-1} \text {. }
$$

(a)

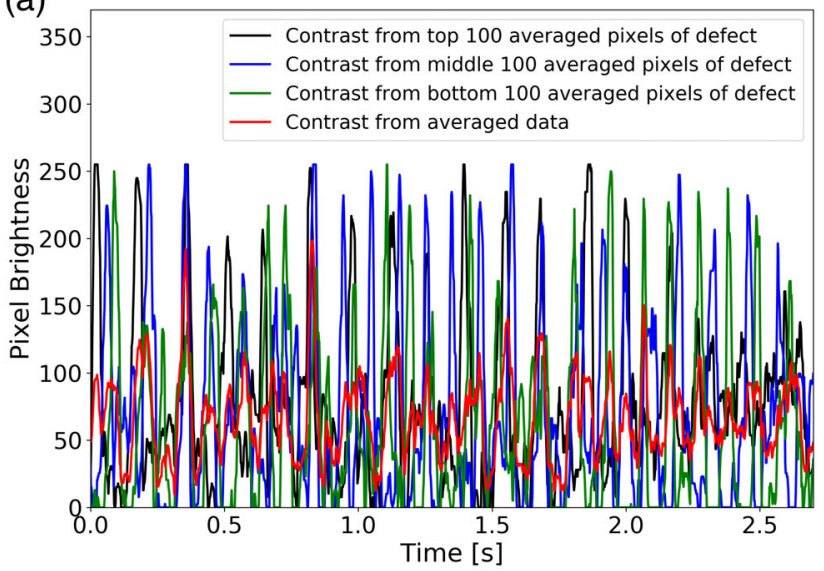

If the analysis is carried out with the apparent velocity $v_{a p p}$ instead, this results in a characteristic frequency of $2.4 \mathrm{~Hz}$ [Figure 26(b)] which is now very close the $1.7 \mathrm{~Hz}$. At least for thin extrudates the shrinking of the extrudate might be the main reason for the discrepancy between the characteristic frequency from pressure and optical analysis. However, this effect should decrease the thicker the sample gets. The die swell and the change from lamellar to plug flow increase the stress on the surface affecting sharkskin.

Stretching and disentanglement of adsorbed chains with bulk chains at the die exit might be another reason leading to a higher characteristic frequencies. For an overview of the different potential origins of the sharkskin instability as a die exit effect please refer to the review papers. ${ }^{1,2}$

\section{CONCLUSIONS}

In this article, a method allowing the identification of the sharkskin instability by its characteristic frequency based on a simple picture analysis is presented. A special focus of this work is the image enhancement. The images are imported in python using the machine learning library OpenCV. To improve the results, the contrast or brightness of the image is adapted. In the case of nonuniform illumination, a Gaussian neighborhood filter significantly improved the analysis. The whole image enhancement is done using built in functions from OpenCV. The results using the optical analysis are in a good agreement compared to the sharkskin

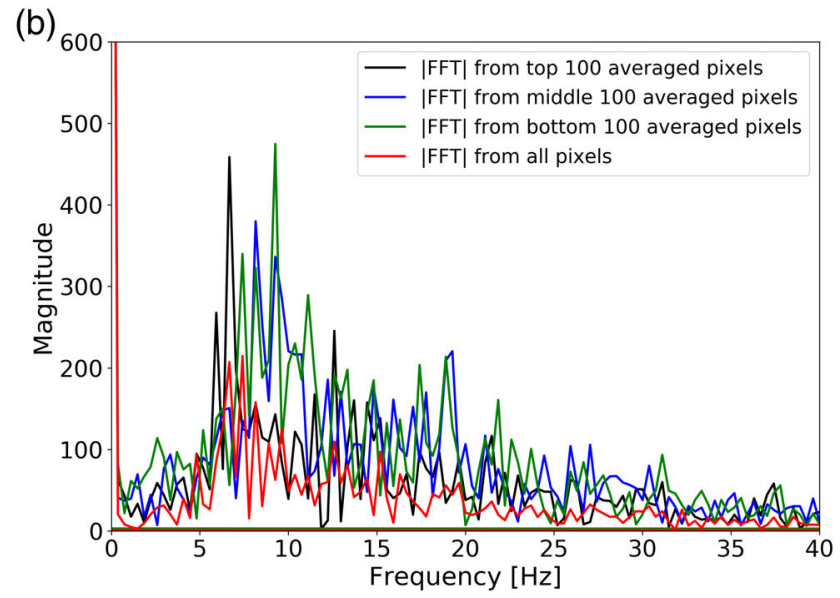

Figure 23. Unfilled SBR extruded through a $L / D=30 / 3$ round hole capillary at $\dot{\gamma}=10 \mathrm{~s}^{-1}$ (a) Time Domain signal representing averaged brightness for different regions, (b) FFT result from optical analysis. [Color figure can be viewed at wileyonlinelibrary.com] 
(a)

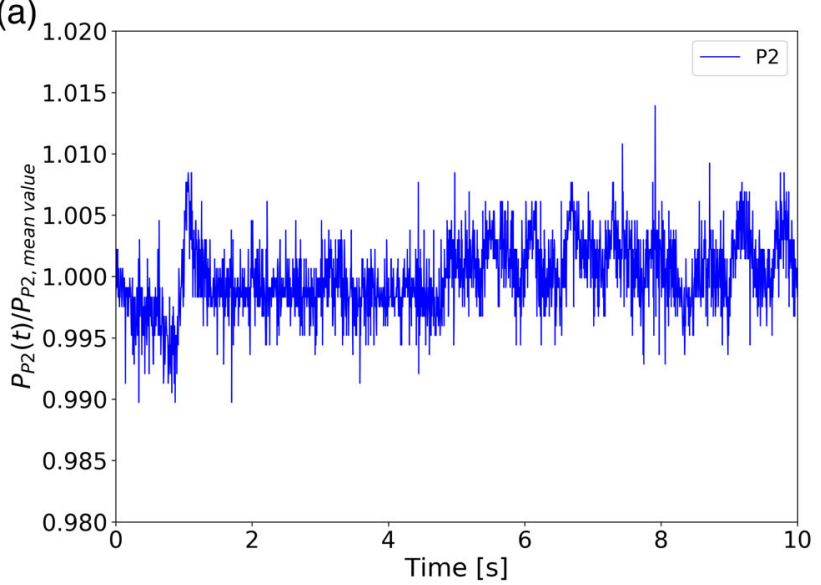

(b)

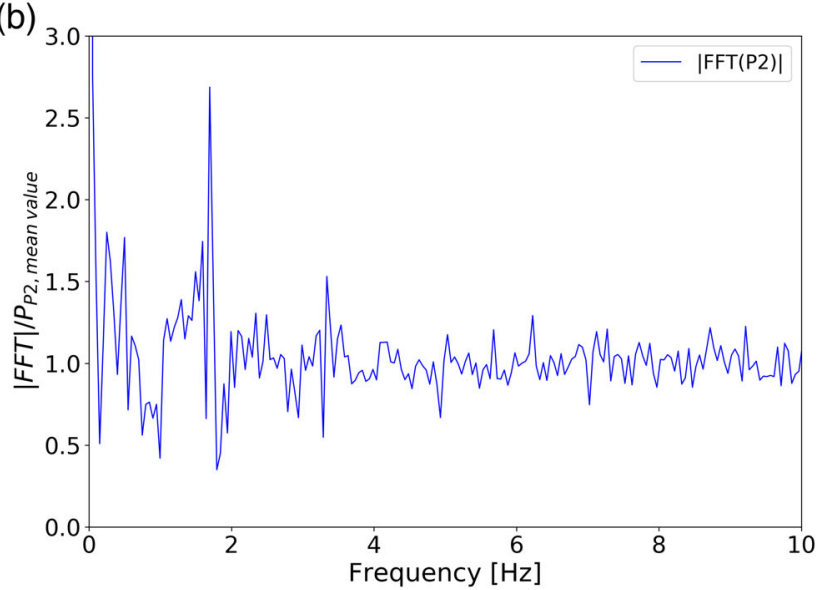

Figure 24. Unfilled SBR extruded at a shear rate of $\dot{\gamma}=33 \mathrm{~s}^{-1}$ (a) Relative pressure from piezoelectric pressure transducer $P 2$, (b) normalised and for readability vertically shifted. [Color figure can be viewed at wileyonlinelibrary.com]

(a)

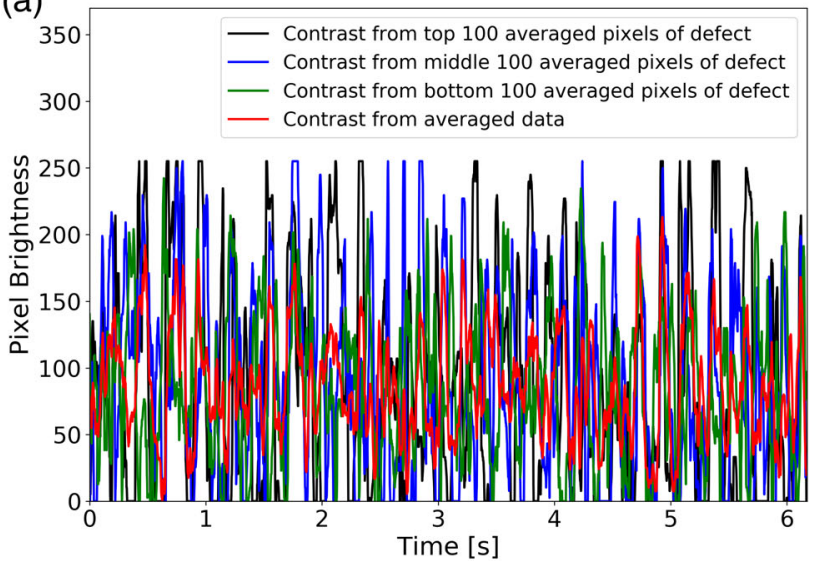

(b)

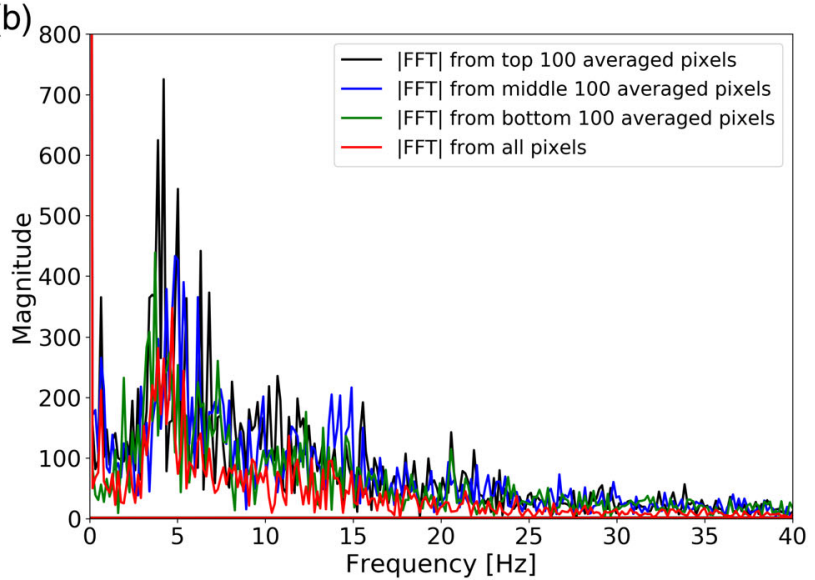

Figure 25. unfilled SBR extruded at a shear rate of $\dot{\gamma}=33 \mathrm{~s}^{-1}$, (a) Pixel brightness versus time after enhancing the image quality with $\alpha=1.2, \beta=-50$, blocksize $=301$, const $=-5$, (b) FFT of optical analysis with a characteristic peak at $4 \mathrm{~Hz}$. [Color figure can be viewed at wileyonlinelibrary.com]

(a)

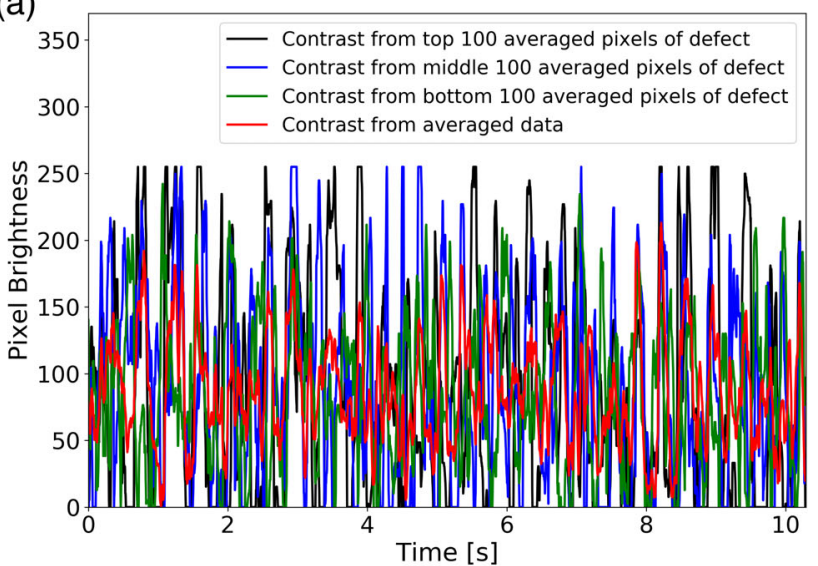

(b)

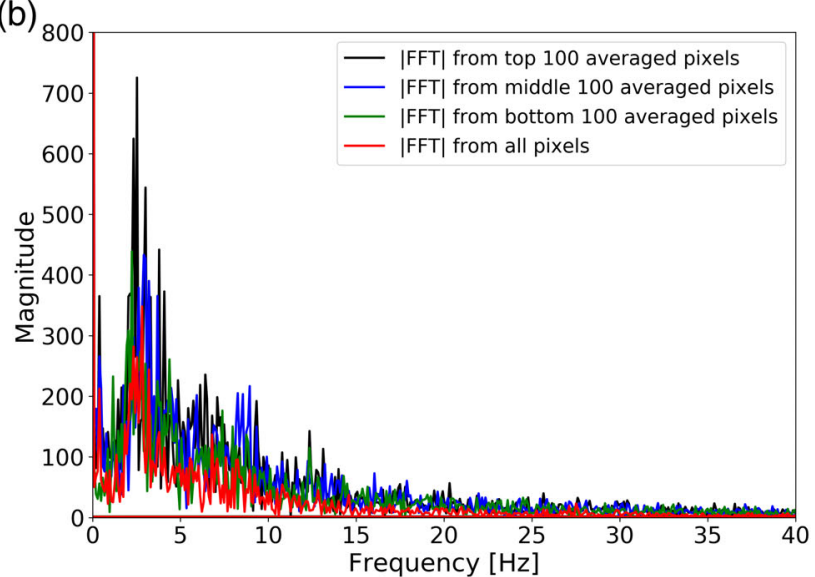

Figure 26. Extrusion velocity calculated from unfilled SBR extrudate, extruded at a shear rate of $\dot{\gamma}=33 \mathrm{~s}^{-1}$ (a) Pixel brightness versus time after enhancing the image quality with $\alpha=1.2, \beta=-50$, blocksize $=301$, const $=-5$, (b) FFT from optical analysis with a characteristic peak at $2.4 \mathrm{~Hz}$. [Color figure can be viewed at wileyonlinelibrary.com] 
option developed by Wilhelm et al. and commercialized by Göttfert. Our results show that the best agreement between the two methods, for most of the cases is obtained if, instead of investigating three different regions, the whole sharkskin instability is considered at once. In this case, distortions and random signals, linked to the image enhancement, which might lead to a periodic signal per region, are averaged out over the whole sample. If the extrudate or instability is however too much deformed, selecting a smaller region to analyze might be better. To improve the accuracy it is important to have a high-resolution camera for high shear rates as the resolution of the FFT is directly related to the size of a pixel. The whole analysis is done using open source Python libraries. This method would also allow us to investigate the transition of the instability when increasing the shear rate as we only need fractions of a second to take a picture instead of $20 \mathrm{~s}-20 \mathrm{~min}$ at a constant pressure to make a measurement. Further investigation of a sample developing the stick slip instability are also planned. The pressure results from the piezoelectric pressure transducers also seem to show that the origin of the instability for the SBR lies within the barrel and not at the die exit, as the relative pressure signal in the piezoelectric pressure transducer $P 1$ (closest to the barrel) is stronger as the relative signal from $P 2$ and $P 3$ located in the middle and the die exit respectively. This results are in contrast to those obtained for polyethylene samples. ${ }^{4}$ This needs to be further investigated. A minimal version of the code can be found on figshare under the DOI: 10.6084/m9.figshare.7993235.

\section{ACKNOWLEDGMENTS}

We thank our industrial partner Goodyear for funding, samples, and equipment. Furthermore, we thank the FNR (Fond National de la Recherche Luxembourg) for the financial funding in the frame of the CORE-PPP project "SLIPEX."

\section{REFERENCES}

1. Agassant, J. F.; Arda, D.; Combeaud, C.; Merten, A.; Muenstedt, H.; Mackley, M.; Robert, L.; Vergnes, B. Int. Polym. Process. 2006, 21(3), 239.
2. Vergnes, B. Int. Polym. Process. 2015, 30(1), 3.

3. Hatzikiriakos, S. G. Prog. Polym. Sci. 2012, 37(4), 624.

4. Palza, H.; Filipe, S.; Naue, I. F. C.; Manfred, W. Polymer. 2010, 51(2), 522.

5. Filipe, S.; Vittorias, I.; Wilhelm, M. Macromol. Mater. Eng. 2008, 293(1), 57.

6. Viloria, M. J.; Valtier, M.; Vergnes, B. J. Rheol. 2017, 61(5), 1085.

7. Tzoganakis, C.; Price, B. C.; Hatzikiriakos, S. G. J. Rheol. 1993, 37(2), 355.

8. Le Gall, F.; Bartos, O.; Davis, J.; Philipp, P. In Polymer Processing Society, European Regional Meeting: Stuttgart, Germany, 1995.

9. Howells, E. R.; Benbow, E. J. J. Trans. Plast. Inst. 1962, 30, 240.

10. Clegg, P. L. Br. Plast. 1957, 30, 535.

11. Bergem, N. In Seventh International Congress on Rheology; Klason, C., Ed.; Göteborg, Sweden, 1976; pp 50-54.

12. Sornberger, G.; Quantin, J. C.; Fajolle, R.; Vergnes, B.; Agassant, J. F. J. Non-Newton Fluid Mech. 1987, 23, 123.

13. Beaufils, P.; Vergnes, B.; Agassant, J. F. Int. Polym. Process. 1989, 4(2), 78.

14. Kalika, D. S.; Morton, M. D. J. Rheol. 1987, 31(8), 815.

15. Naue, I. F. C.; Kádár, R.; Wilhelm, M. M. Macromol. Mater. Eng. 2015, 300(11), 1141.

16. Naue, I. F. C. Ph.D. thesis, Institut für Technische Chemie und Polymerchemie, 2013.

17. Palza, H.; Naue, I. F. C.; Filipe, S.; Becker, A.; Sunder, J.; Göttfert, A.; Manfred, W. KGK. 2010, 63(10), 456.

18. Smith S. W. The Scientist and Engineer's Guide to Digital Signal Processing. California Technical Pub: San Diego, Calif, 1997.

19. Bradski, G. DDJ, 2000, 120, 122.

20. Palza, H.; Naue, I. F. C.; Manfred, W. Macromol. Rapid Commun. 2009, 30(21), 1799. 